\title{
On the eigenvalues of Cayley graphs on the symmetric group generated by a complete multipartite set of transpositions
}

\author{
Filippo Cesi
}

Received: 11 February 2009 / Accepted: 2 November 2009 / Published online: 24 November 2009

(C) Springer Science+Business Media, LLC 2009

\begin{abstract}
Given a finite simple graph $\mathscr{G}$ with $n$ vertices, we can construct the Cayley graph on the symmetric group $S_{n}$ generated by the edges of $\mathscr{G}$, interpreted as transpositions. We show that, if $\mathscr{G}$ is complete multipartite, the eigenvalues of the Laplacian of Cay $(\mathscr{G})$ have a simple expression in terms of the irreducible characters of transpositions and of the Littlewood-Richardson coefficients. As a consequence, we can prove that the Laplacians of $\mathscr{G}$ and of Cay $(\mathscr{G})$ have the same first nontrivial eigenvalue. This is equivalent to saying that Aldous's conjecture, asserting that the random walk and the interchange process have the same spectral gap, holds for complete multipartite graphs.
\end{abstract}

Keywords Cayley graphs · Laplacian · Symmetric group · Littlewood-Richardson rule $\cdot$ Spectral gap $\cdot$ Interchange process

\section{Introduction}

Let $\mathscr{G}=(V(\mathscr{G}), E(\mathscr{G}))$ be a finite graph with $V(\mathscr{G})=\{1,2, \ldots, n\}$. $\mathscr{G}$ is always assumed to be simple, i.e., without multiple edges and loops, and undirected. The Laplacian of $\mathscr{G}$ is the $n \times n$ matrix $\Delta_{\mathscr{G}}:=D-A$, where $A$ is the adjacency matrix of $\mathscr{G}$, and $D=\operatorname{diag}\left(d_{1}, \ldots, d_{n}\right)$ with $d_{i}$ denoting the degree of the vertex $i$. Since $\Delta \mathscr{G}$ is symmetric and positive semidefinite, its eigenvalues are real and nonnegative and can be ordered as $0=\lambda_{1} \leq \lambda_{2} \leq \cdots \leq \lambda_{n}$. There is an extensive literature dealing with bounds on the distribution of the eigenvalues and consequences of these bounds.

\footnotetext{
F. Cesi $(\bowtie)$

Dipartimento di Fisica, Università di Roma "La Sapienza", Rome, Italy

e-mail: filippo.cesi@roma1.infn.it

F. Cesi

SMC, INFM-CNR, Rome, Italy
} 
We refer the reader to [2, 4, 9] for a general introduction to the subject. If (and only if) $\mathscr{G}$ is connected, the second eigenvalue $\lambda_{2}$ is positive and is of particular importance for several applications. The Laplacian $\Delta \mathscr{G}$ can be viewed as the generator ${ }^{1}$ of a continuous-time random walk on $V$, whose invariant measure is the uniform measure $u$ on $V$. In this respect, $\lambda_{2}$ is the inverse of the "relaxation time" of the random walk, a quantity related to the speed of convergence to the invariant measure in $L^{2}(V, u)$ sense. $\lambda_{2}$ is also called the spectral gap of $\Delta \mathscr{G}$.

There is a natural way to associate a Cayley graph to $\mathscr{G}$. Any edge $e=\{i, j\}$ in $E(\mathscr{G})$ (and, more generally, any pair $\{i, j\}$ of elements of $V(\mathscr{G})$ ) can be identified with a transposition $(i j)$ of the symmetric group $S_{n}$. Consider then the Cayley graph with vertex set equal to $S_{n}$ and edges given by $(\pi, \pi e)$, where $\pi$ is a permutation of $S_{n}$ and $e \in E(\mathscr{G})$. We let, for simplicity,

$$
\operatorname{Cay}(\mathscr{G}):=\operatorname{Cay}\left(S_{n}, E(\mathscr{G})\right) .
$$

The Laplacian of Cay $(\mathscr{G})$ is again the generator of a continuous-time Markov chain called the interchange process on $V$. It can be described as follows: each site of $V$ is occupied by a particle of a different color, and for each edge $\{i, j\} \in E(\mathscr{G})$, at rate 1 , the particles at vertices $i$ and $j$ are exchanged.

It is easy to show (it follows from (3.14) and (3.15), but there are simpler and more direct proofs) that the spectrum of $\Delta_{\mathscr{G}}$ is a subset of the spectrum of $\Delta_{\text {Cay }(\mathscr{G})}$. By consequence,

$$
\lambda_{2}(\Delta \mathscr{G}) \geq \lambda_{2}\left(\Delta_{\text {Cay }(\mathscr{G})}\right) .
$$

Being an $n ! \times n !$ matrix, in general the Laplacian of Cay $(\mathscr{G})$ has many more eigenvalues than the Laplacian of $\mathscr{G}$. Nevertheless, a neat conjecture due to David Aldous [1] states, equivalently:

Aldous's conjecture (v.1) If $\mathscr{G}$ is a finite connected simple graph, then

$$
\lambda_{2}(\Delta \mathscr{G})=\lambda_{2}\left(\Delta_{\text {Cay }(\mathscr{G})}\right) .
$$

Aldous's conjecture (v.2) If $\mathscr{G}$ is a finite connected simple graph, then the random walk and the interchange process on $\mathscr{G}$ have the same spectral gap.

Version 1 also appears in [8], under the extra assumption of $\mathscr{G}$ being bipartite. Aldous's conjecture comes in a third flavor, which originates from the analysis of the representations of the symmetric group. ${ }^{2}$ From this point of view the Laplacian $\Delta_{\mathscr{G}}$ corresponds to the $n$-dimensional defining representation of $S_{n}$, whose irreducible components are the trivial representation and the representation associated with the partition $(n-1,1)$. On the other hand, the Laplacian of the Cayley graph Cay $(\mathscr{G})$ is associated to the right regular representation, which contains all irreducible representations. Let $\alpha=\left(\alpha_{1}, \ldots, \alpha_{r}\right)$ be a partition of $n$ and denote with $\mathbf{T}^{\alpha}$ the irreducible

\footnotetext{
${ }^{1}$ Or minus the generator, depending on the preferred sign convention.

${ }^{2}$ This will be explained in greater detail in Sects. 2 and 3 .
} 
representation of $S_{n}$ which corresponds to the partition $\alpha$. Let also $\lambda_{\max }(\alpha)$ be the maximum eigenvalue of the matrix

$$
\sum_{e=\{i, j\} \in E(\mathscr{G})} \mathbf{T}^{\alpha}(e) .
$$

The trivial representation, which corresponds to the trivial partition $\alpha=(n)$, is thus contained with multiplicity one in both the defining and right regular representations, and clearly $\lambda_{\max }((n))=|E(\mathscr{G})|$. This accounts for the fact that the first eigenvalue of both $\Delta_{\mathscr{G}}$ and $\Delta_{\mathrm{Cay}(\mathscr{G})}$ is null. By consequence, one finds (see Sect. 3) that Aldous's conjecture can be restated as follows:

Aldous's conjecture (v.3) If $\mathscr{G}$ is a finite connected simple graph, then

$$
\lambda_{\max }(\alpha) \leq \lambda_{\max }((n-1,1))
$$

for each nontrivial partition $\alpha$ of $n$, i.e., for each partition $\alpha \neq(n)$.

Aldous's conjecture has been proven for star-graphs in [7] and for complete graphs in [6]. A major progress was made in [10] (similar results were reobtained in [13]), where a rather general technique was developed, which can be used to prove the conjecture for trees (with weighted edges) and a few other cases. Without entering into the details, we mention that this technique is useful for classes of graphs whose spectral gap "tends to decrease" when a new site, and relative edges, are added to a preexisting graph. This is indeed the case of trees, since it is not too difficult to prove that adding a leaf with its relative edge cannot increase the spectral gap. Using this approach, Aldous's conjecture has been recently proven [14, 16] for hypercubes asymptotically, i.e., in the limit as the side length of the cube tends to infinity.

The main result of the present paper is the proof of Aldous's conjecture for complete multipartite graphs (Theorem 3.1). These are graphs such that it is possible to write the vertex set of $\mathscr{G}$ as a disjoint union

$$
V(\mathscr{G})=\{1, \ldots, n\}=N_{1} \cup \cdots \cup N_{p}
$$

in such a way that $\{i, j\}$ is an edge if and only if $i$ and $j$ belong to distinct $N_{k}$ 's. The approach we follow, similar in spirit to [6], is group theoretical.

The plan of the paper is as follows. After recalling a few standard facts on the representation theory of the symmetric group in Sect. 2, we discuss the relationship between the Laplacian of Cayley graphs and the irreducible representations of $S_{n}$ in Sect. 3. In Sect. 4 the proof of our main result is outlined in the case of bipartite graphs. Most of the relevant ideas are discussed in this section. Section 5 contains a detailed proof of the general multipartite case. One of the key technical ingredients is the identification of the Littlewood-Richardson tableau with minimal content, among all tableaux which appear in the decomposition of a tensor product of representations of $S_{n}$ (Lemma 5.11). This aspect is discussed in Sect. 6.

After this paper was completed, a beautiful proof of Aldous's conjecture has been found by Caputo, Liggett, and Richthammer [3], which holds for arbitrary graphs (including weighted graphs). Their approach is based on a subtle mapping, reminiscent 
of the star-triangle transformation used in electric networks, which allows a recursive proof.

\section{The irreducible representations of $S_{n}$}

We recall here a few well-known facts from the representation theory of the symmetric group. The main purpose is to establish our notation. Standard references for this section are, for instance, [5] for general representation theory and [12, 15] for the symmetric group.

Given a positive integer $n$, a composition (resp. a weak composition) of $n$ is a sequence $\alpha=\left(\alpha_{1}, \alpha_{2}, \alpha_{3}, \ldots\right)$ of positive (resp. nonnegative) integers such that $\sum_{i=1}^{\infty} \alpha_{i}=n$. Since there is only a finite number of nonzero terms, one can either consider the whole infinite sequence or just the finite sequence obtained by dropping all trailing zeros which appear after the last nonzero element. We define the length of $\alpha$ as the position of the last nonzero element in $\alpha$, so if the length of $\alpha$ is $r$, we write

$$
\alpha=\left(\alpha_{1}, \ldots, \alpha_{r}\right)=\left(\alpha_{1}, \ldots, \alpha_{r}, 0,0,0, \ldots\right) .
$$

We also let $|\alpha|:=\sum_{i} \alpha_{i}$, while, for an arbitrary set $S,|S|$ stands, as usual, for the cardinality of $S$. A partition of $n$ is a nonincreasing composition of $n$. We write $\alpha \models n$ if $\alpha$ is a composition of $n, \alpha \stackrel{\text { }}{=} n$ if $\alpha$ is a weak composition on $n$, and $\alpha \vdash n$ if $\alpha$ is a partition of $n$.

We introduce a componentwise partial order in the set of all finite sequences of integers: we write $\alpha \leq \beta$ if $\alpha_{i} \leq \beta_{i}$ for each $i$. If $\alpha, \beta$ are partitions (compositions, weak compositions), we can define the component-by-component sum $\alpha+\beta$ which is still a partition (composition, weak composition). If $\alpha \leq \beta$, the difference $\beta-\alpha$ is a weak composition of $|\beta|-|\alpha|$.

The Young diagram of a partition $\alpha$ of $n$ is a graphical representation of $\alpha$ as a collection of $n$ boxes arranged in left-justified rows, with the $i$ th row containing $\alpha_{i}$ boxes. For instance,

$$
(6,4,1)=\begin{array}{|l|l|l|l|l|l|}
\hline & & & & & \\
\hline & & & & \multicolumn{3}{|l}{} \\
\hline
\end{array}
$$

We do not distinguish between a partition and its associated Young diagram. If the integer $k$ appears $m$ times in the partition $\alpha$, we may simply write $k^{m}$, so, for instance,

$$
(5,5,4,2,2,2,1,1)=\left(5^{2}, 4,2^{3}, 1^{2}\right) .
$$

Given a Young diagram $\alpha$, the conjugate (or transpose) diagram is the diagram, denoted with $\alpha^{\prime}$, obtained from $\alpha$ by "exchanging rows and columns," for example,
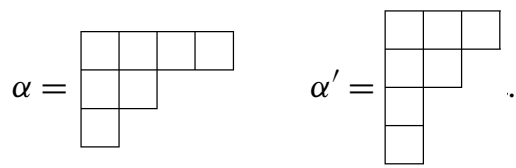
The elements of $\alpha^{\prime}$ are given by

$$
\alpha_{s}^{\prime}:=\left|\left\{j: \alpha_{j} \geq s\right\}\right|
$$

Let $\operatorname{Irr}\left(S_{n}\right)$ be the set of all equivalence classes of irreducible representations ${ }^{3}$ of $S_{n}$. There is a one-to-one correspondence between $\operatorname{Irr}\left(S_{n}\right)$ and the set of all partitions of $n$. We denote with $[\alpha]$ the class of irreducible representations of $S_{n}$ corresponding to the partition $\alpha$, and with $f_{\alpha}$ the dimension or degree of the representation. For simplicity, we write $\left[\alpha_{1}, \ldots, \alpha_{r}\right]$ instead of $\left[\left(\alpha_{1}, \ldots, \alpha_{r}\right)\right]$. It is sometimes notationally convenient to refer to a specific choice of a representative in the class $[\alpha]$. We denote this choice with $\mathbf{T}^{\alpha}$. Hence $\mathbf{T}^{\alpha}$ is a group homomorphism

$$
\mathbf{T}^{\alpha}: S_{n} \mapsto G L\left(f_{\alpha}, \mathbb{C}\right) \quad \alpha \vdash n
$$

Since every representation of a finite group is equivalent to a unitary representation, we can assume (if useful) that $\mathbf{T}^{\alpha}(\pi)$ is a unitary matrix for each $\pi \in S_{n}$. Every representation $\mathbf{Y}$ of $S_{n}$ can be written, modulo equivalence, as a direct sum of the $\mathbf{T}^{\alpha}$,

$$
\mathbf{Y} \cong \bigoplus_{\alpha \vdash n} c_{\alpha} \mathbf{T}^{\alpha}
$$

A fundamental quantity associated to a representation $\mathbf{Y}$ of a finite group $G$ is the character of $\mathbf{Y}$, which we denote by $\chi^{\mathbf{Y}}$, and is defined as

$$
\begin{aligned}
\chi^{\mathbf{Y}}: G & \mapsto \mathbb{C} \\
: g & \rightarrow \operatorname{tr} \mathbf{Y}(g) .
\end{aligned}
$$

Two representations are equivalent if and only if they have the same characters, so, going back to $G=S_{n}$, we can choose an arbitrary representative $\mathbf{T}^{\alpha}$ in the class $[\alpha]$ and define $\chi^{\alpha}:=\chi^{\mathbf{T}^{\alpha}}$. The set $\left\{\chi^{\alpha}: \alpha \vdash n\right\}$ is the set of irreducible characters of $S_{n}$.

If $H$ is a subgroup of $G$, we denote with $\mathbf{Y} \downarrow_{H}^{G}$ the restriction of the representation $\mathbf{Y}$ to $H$. Even if $\mathbf{Y}$ is an irreducible representation of $G$, the restriction $\mathbf{Y} \downarrow_{H}^{G}$ is in general a reducible representation of $H$. By consequence, there exists a collection of nonnegative integers $\left(c_{T}\right)$ such that

$$
\mathbf{Y} \downarrow_{H}^{G}=\bigoplus_{\mathbf{T} \in \operatorname{Irr}(H)} c_{T} \mathbf{T} .
$$

When $G=S_{n}$ and $H$ is a Young subgroup $S_{(j, k)}$ (see Sect. 5), the coefficients $c_{T}$ are called the Littlewood-Richardson coefficients. ${ }^{4}$

\footnotetext{
${ }^{3}$ In this paper, by "representation" we mean a finite-dimensional representation over the field of complex numbers.

${ }^{4}$ The Littlewood-Richardson coefficients are often equivalently (thanks to Frobenius reciprocity) defined in terms of an induced representation.
} 


\section{Eigenvalues of Cayley graphs and representations of $S_{n}$}

We illustrate how, when studying the eigenvalues of the Laplacian of Cayley graphs, one is (almost forcibly) led to consider the irreducible representations of the symmetric group. In this way we can show that version 3 of Aldous's conjecture is equivalent to versions 1 and 2. The material of this section is more or less standard and overlaps with Sect. 4 of [6].

Given a finite set $S=\left\{s_{1}, \ldots, s_{n}\right\}$, we denote with $\mathbb{C} S$ the $n$-dimensional vector space which consists of all formal complex linear combinations of the symbols $\left\{s_{1}\right\}, \ldots,\left\{s_{n}\right\}$, and with $\mathbb{C}^{S}$ the vector space of all functions $f: S \mapsto \mathbb{C}$. $\mathbb{C}^{S}$ is naturally isomorphic to $\mathbb{C} S$ under the correspondence

$$
f \longleftrightarrow \sum_{i=1}^{n} f(i)\left\{s_{i}\right\}
$$

Any left action $(g, s) \rightarrow g s$ of a finite group $G$ on $S$ defines a representation $\mathbf{Y}$ of $G$ on $\mathbb{C} S$ given by

$$
\mathbf{Y}(g)\left(\sum_{i=1}^{n} a_{i}\left\{s_{i}\right\}\right):=\sum_{i=1}^{n} a_{i}\left\{g s_{i}\right\} \quad g \in G .
$$

One can, equivalently, interpret $\mathbf{Y}$ as a representation on $\mathbb{C}^{S}$, in which case we have $\mathrm{e}^{5}$

$$
[\mathbf{Y}(g) f](s):=f\left(g^{-1} s\right) \quad g \in G, f \in \mathbb{C}^{S} .
$$

$\mathbb{C} G$ is the (complex) group algebra of $G$. Any representation $\mathbf{Y}$ of $G$ extends to a representation of $\mathbb{C} G$ by letting

$$
\mathbf{Y}\left(\sum_{g \in G} a_{g} g\right):=\sum_{g \in G} a_{g} \mathbf{Y}(g) \quad a_{g} \in \mathbb{C} .
$$

Let then $\mathscr{G}$ be a finite graph with $V(\mathscr{G})=\{1, \ldots, n\}$. The defining representation of $S_{n}$, which we denote by $\mathbf{D}$, acts on $\mathbb{C} V=\mathbb{C}\{1, \ldots, n\}$ as

$$
\mathbf{D}(\pi)\left(\sum_{i=1}^{n} a_{i}\{i\}\right)=\sum_{i=1}^{n} a_{i}\{\pi(i)\} \quad \pi \in S_{n} .
$$

The matrix elements of $\mathbf{D}(\pi)$ in this basis are given by

$$
[\mathbf{D}(\pi)]_{i j}= \begin{cases}1 & \text { if } j=\pi^{-1}(i), \\ 0 & \text { otherwise }\end{cases}
$$

\footnotetext{
${ }^{5}$ With a slight abuse of notation we use the same symbol $\mathbf{Y}$ since the two representations are equivalent under (3.1). 
The action of $\mathbf{D}$ on the space $\mathbb{C}^{V}$ is $[\mathbf{D}(\pi) f](i)=f\left(\pi^{-1}(i)\right)$, i.e., $\mathbf{D}(\pi) f=f \circ \pi^{-1}$. If $\pi$ is a transposition, $\pi=(k l)$, we have

$$
[\mathbf{D}((k l)) f](i):= \begin{cases}f(k) & \text { if } i=l, \\ f(l) & \text { if } i=k, \\ f(i) & \text { if } i \neq k, l .\end{cases}
$$

Hence, under the identification of edges with transpositions of $S_{n}$

$$
E(\mathscr{G}) \ni e=\{i, j\} \longrightarrow(i j) \in\left\{\pi \in S_{n}: \pi \text { is a transposition }\right\},
$$

we can write

$$
\begin{aligned}
(\Delta \mathscr{G} f)(i) & =\sum_{j:(i j) \in E(\mathscr{G})}[f(i)-f(j)] \\
& =\sum_{j: e=(i j) \in E(\mathscr{G})}[f(i)-\mathbf{D}(e) f(i)]=\sum_{e \in E(\mathscr{G})}[f(i)-\mathbf{D}(e) f(i)],
\end{aligned}
$$

where, in the last term, we have included the null contribution of those edges with both endpoints different from $i$. The reason is that we can now rewrite (3.5) in operator form. If denote with $I_{n}$ the identity operator acting on an $n$-dimensional vector space, we have

$$
\Delta_{\mathscr{G}}=|E(\mathscr{G})| I_{n}-\sum_{e \in E(\mathscr{G})} \mathbf{D}(e)=|E(\mathscr{G})| I_{n}-\mathbf{D}\left(\sum_{e \in E(\mathscr{G})} e\right)
$$

where, in view of correspondence (3.4), $\sum_{e \in E(\mathscr{G})} e$ can be considered an element of the group algebra $\mathbb{C} S_{n}$, and, in the last equality, we have used the linear extension of D to a representation of $\mathbb{C} S_{n}$. Given a finite graph $\mathscr{G}$, we define

$$
W(\mathscr{G}):=\sum_{e \in E(\mathscr{G})} e \in \mathbb{C} S_{n}
$$

and rewrite (3.6) as

$$
\Delta \mathscr{G}=|E(\mathscr{G})| I_{n}-\mathbf{D}[W(\mathscr{G})] .
$$

A relationship for the corresponding eigenvalues trivially follows:

$$
\lambda_{i}(\Delta \mathscr{G})=|E(\mathscr{G})|-\lambda_{n-i}(\mathbf{D}[W(\mathscr{G})]) \quad i=1, \ldots, n .
$$

We remark that, in the more general case of a weighted graph with edge weights $\left(w_{e}\right)_{e \in E(\mathscr{G})}$, identities (3.8) and (3.9) remain valid as long as one uses the "correct definition" of $W(\mathscr{G})$ as $W(\mathscr{G}):=\sum_{e \in E(\mathscr{G})} w_{e} e$.

We can associate to the graph $\mathscr{G}$ the Cayley graph $\operatorname{Cay}\left(S_{n}, E(\mathscr{G})\right)$ with vertex set $S_{n}$, where $n$ is the cardinality of $V(\mathscr{G})$, and edge set given by

$$
\left\{(\pi, \pi e): \pi \in S_{n}, e=(i j) \in E(\mathscr{G})\right\} .
$$


Since each transposition coincides with its inverse, this Cayley graph is undirected. We let, for simplicity, $\operatorname{Cay}(\mathscr{G}):=\operatorname{Cay}\left(S_{n}, E(\mathscr{G})\right)$. If we denote with $\mathbf{R}$ the right regular representation of $S_{n}$ which acts on $S_{n}$ and on $\mathbb{C}^{S_{n}}$ respectively as ${ }^{6}$

$$
\begin{aligned}
& \mathbf{R}(\pi) \pi^{\prime}=\pi^{\prime} \pi^{-1} \quad \pi, \pi^{\prime} \in S_{n}, \\
& {[\mathbf{R}(\pi) f]\left(\pi^{\prime}\right)=f\left(\pi^{\prime} \pi\right) \quad f: S_{n} \mapsto \mathbb{C},}
\end{aligned}
$$

we can proceed as in (3.5) and obtain

$$
\left(\Delta_{\mathrm{Cay}(\mathscr{G})} f\right)(\pi)=\sum_{e \in E(\mathscr{G})}[f(\pi)-f(\pi e)]=\sum_{e \in E(\mathscr{G})}[f(\pi)-\mathbf{R}(e) f(\pi)] .
$$

Identities (3.8) and (3.9) become, for the Cayley graph,

$$
\begin{aligned}
\Delta_{\text {Cay }(\mathscr{G})} & =|E(\mathscr{G})| I_{n !}-\mathbf{R}[W(\mathscr{G})] \\
\lambda_{i}\left(\Delta_{\mathrm{Cay}(\mathscr{G})}\right) & =|E(\mathscr{G})|-\lambda_{n !-i}(\mathbf{R}[W(\mathscr{G})]) \quad i=1, \ldots, n ! .
\end{aligned}
$$

The right regular representation $\mathbf{R}$ is equivalent to the left regular representation (under the change of basis $\pi \rightarrow \pi^{-1}$ ) and can be written as a direct sum of all irreducible representations, each appearing with a multiplicity equal to its dimension,

$$
[\mathbf{R}]=\bigoplus_{\alpha \vdash n} f_{\alpha}[\alpha]
$$

By consequence, the spectrum of $\mathbf{R}[W(\mathscr{G})]$ can be written as ${ }^{7}$

$$
\operatorname{spec} \mathbf{R}[W(\mathscr{G})]=\bigcup_{\alpha \vdash n} \operatorname{spec} \mathbf{T}^{\alpha}[W(\mathscr{G})] .
$$

The (trivial) one-dimensional identity representation $\mathbf{T}^{(n)}=I_{1}$, corresponding to the partition $(n)$, appears in this decomposition exactly once, and we have $\mathbf{T}^{(n)}[W(\mathscr{G})]=$ $|E(\mathscr{G})| \cdot I_{1}$; thus its unique eigenvalue is equal to $|E(\mathscr{G})|$, which accounts for the fact that $\lambda_{1}\left(\Delta_{\text {Cay }(\mathscr{G})}\right)=0$. If $\mathscr{G}$ is connected, the set $E(\mathscr{G})$, considered as a set of transpositions, generates $S_{n}$, and hence $\operatorname{Cay}(\mathscr{G})$ is also connected, and $\lambda_{1}$ is the unique null eigenvalue of $\Delta_{\text {Cay }(\mathscr{G})}$. In any case, letting

$$
\lambda_{\max }[\alpha, W(\mathscr{G})]:=\max \operatorname{spec} \mathbf{T}^{\alpha}[W(\mathscr{G})]=\lambda_{f_{\alpha}}\left(\mathbf{T}^{\alpha}[W(\mathscr{G})]\right),
$$

we have, for what concerns the second eigenvalue of the Cayley graph,

$$
\lambda_{2}\left(\Delta_{\mathrm{Cay}(\mathscr{G})}\right)=|E(\mathscr{G})|-\max _{\alpha \vdash n, \alpha \neq(n)} \lambda_{\max }[\alpha, W(\mathscr{G})] .
$$

\footnotetext{
${ }^{6}$ The right regular representation is a left action, like every representation.

${ }^{7}$ To get the correct multiplicities of the eigenvalues, one must include the coefficients $f_{\alpha}$ and interpret the union over $\alpha$ as a disjoint union of multisets.
} 
On the other hand, the defining representation can be decomposed as $[\mathbf{D}]=[n] \oplus$ $[n-1,1]$, which implies

$$
\lambda_{2}(\Delta \mathscr{G})=|E(\mathscr{G})|-\lambda_{\max }[(n-1,1), W(\mathscr{G})] .
$$

The main result of paper is the following:

Theorem 3.1 If $\mathscr{G}$ is a complete multipartite graph with $n$ vertices, we have

$$
\lambda_{\max }[\alpha, W(\mathscr{G})] \leq \lambda_{\max }[(n-1,1), W(\mathscr{G})]
$$

for all irreducible representations $[\alpha]$ of $S_{n}$ with $[\alpha] \neq[n]$.

From (3.14), (3.15), and Theorem 3.1 it follows that:

Corollary 3.2 If $\mathscr{G}$ is a complete multipartite graph, then Aldous's conjecture holds, that is,

$$
\lambda_{2}\left(\Delta_{\mathrm{Cay}}(\mathscr{G})\right)=\lambda_{2}(\Delta \mathscr{G}) .
$$

\section{Outline of the proof in the bipartite case}

We briefly sketch in this section the proof of Theorem 3.1 in the bipartite case, which requires less notation than the more general multipartite case but illustrates most of the relevant ideas. The general case can be treated by relatively standard induction. All missing details will be found in later sections.

We start with a well-known fact [6] about the complete graph $K_{n}$. Given an irreducible representation $[\alpha]$ of $S_{n}$, corresponding to the partition $\alpha=\left(\alpha_{1}, \ldots, \alpha_{r}\right)$, we consider the normalized character on the sum of all transpositions

$$
q_{\alpha}:=\frac{n(n-1)}{2 f_{\alpha}} \chi^{\alpha}(e) \quad \alpha \vdash n,
$$

where $e$ is an arbitrary transposition of $S_{n}$. In the case of transpositions, Frobenius formulas for the irreducible characters take the simple form [11]

$$
q_{\alpha}=\frac{1}{2} \sum_{i=1}^{\infty} \alpha_{i}\left[\alpha_{i}-(2 i-1)\right]=\frac{1}{2} \sum_{i=1}^{r} \alpha_{i}\left[\alpha_{i}-(2 i-1)\right],
$$

where $r$ is the length of $\alpha$. We use expression (4.1) as a definition of $q_{\alpha}$ when $\alpha$ is, more generally, a weak composition of $n$, even though, when $\alpha$ is not a partition, the quantity $q_{\alpha}$ has no significance associated to an irreducible representation of $S_{n}$. A simple application of the Schur's lemma yields the following result (see [6, Lemma 5] for a more general statement where arbitrary conjugacy classes are considered).

Proposition 4.1 If $\mathbf{T}^{\alpha}$ is an irreducible representation of $S_{n}$ corresponding to the partition $\alpha \vdash n$, then

$$
\mathbf{T}^{\alpha}\left[W\left(K_{n}\right)\right]=q_{\alpha} I_{f_{\alpha}} .
$$


Table 1 Eigenvalues of $\mathbf{T}^{(4,2,1)}\left[W\left(K_{4,3}\right)\right]$

\begin{tabular}{llrllr}
\hline$\alpha=(4,2,1)$ & \multicolumn{2}{l}{$q_{\alpha}-q_{\beta}-q_{\gamma}$} & & & \\
\hline$\beta$ & $\gamma$ & $\lambda$ & $\beta$ & $\gamma$ & $\lambda$ \\
\hline$(4)$ & $(2,1)$ & -3 & $(3,1)$ & $(3)$ & -2 \\
$(3,1)$ & $(2,1)$ & 1 & $(3,1)$ & $\left(1^{3}\right)$ & 4 \\
$(2,2)$ & $(3)$ & 0 & $(2,2)$ & $(2,1)$ & 3 \\
$(2,1,1)$ & $(3)$ & 2 & $(2,1,1)$ & $(2,1)$ & 5 \\
\hline
\end{tabular}

Let then $n=j+k$ with $j, k$ two positive integers and consider the complete bipartite graph $K_{j, k}$ with vertex set $\{1, \ldots, n\}$ and edges $\left\{i, i^{\prime}\right\}$ with $i \leq j$ and $i^{\prime}>j$. Since the complement of $K_{j, k}$ is given by

$$
\bar{K}_{j, k}=K_{j} \cup K_{k},
$$

using Proposition 4.1, one can prove (see Proposition 5.3) that the eigenvalues of $\mathbf{T}^{\alpha}\left[W\left(K_{j, k}\right)\right]$ have the form

$$
q_{\alpha}-q_{\beta}-q_{\gamma}
$$

where $\beta$ is a partition of $j$, and $\gamma$ is a partition of $k$, subject to the condition that the Littlewood-Richardson coefficient $c_{\beta, \gamma}^{\alpha}$ is positive. The reason for this is that the irreducible representation $[\alpha]$ of $S_{n}$ is no longer irreducible when restricted to the Young subgroup $S_{(j, k)} \cong S_{j} \times S_{k}$, but it is a direct sum of irreducible components

$$
[\alpha] \downarrow_{S_{(j, k)}}^{S_{n}}=\bigoplus_{\beta \vdash j, \gamma \vdash k} c_{\beta, \gamma}^{\alpha}[\beta] \otimes[\gamma] .
$$

If one is interested in keeping track of multiplicities, each pair $(\beta, \gamma)$ appearing in (4.3) contributes with a multiplicity equal to $c_{\beta, \gamma}^{\alpha} f_{\beta} f_{\gamma}$. For example, using the Littlewood-Richardson rule (see Sect. 6), we find the decomposition

$$
\begin{aligned}
{[4,2,1] \downarrow_{S_{(4,3)}}^{S_{7}}=} & {[4] \otimes[2,1] \oplus[3,1] \otimes[3] \oplus 2[3,1] \otimes[2,1] \oplus[3,1] \otimes\left[1^{3}\right] } \\
& \oplus\left[2^{2}\right] \otimes[3] \oplus\left[2^{2}\right] \otimes[2,1] \oplus\left[2,1^{2}\right] \otimes[3] \oplus\left[2,1^{2}\right] \otimes[2,1] .
\end{aligned}
$$

This, in turn, determines that the eigenvalues of $\mathbf{T}^{(4,2,1)}\left[W\left(K_{4,3}\right)\right]$ are those given in Table 1 . Thus $\lambda_{\max }\left((4,2,1), W\left[K_{4,3}\right]\right)=5$.

We say that the pair $(\beta, \gamma)$ is $\alpha$-admissible if $c_{\beta, \gamma}^{\alpha}>0$, and we define

$$
B_{j, k}^{\alpha}:=\max _{\alpha \text {-admissible }(\beta, \gamma)} q_{\alpha}-q_{\beta}-q_{\gamma},
$$

so that

$$
\lambda_{\max }\left[\alpha, W\left(K_{j, k}\right)\right]=B_{j, k}^{\alpha} .
$$

In general, given $\alpha \vdash n$ and $\beta \vdash j$, there are several different $\gamma \vdash k$ such that $(\beta, \gamma)$ is $\alpha$-admissible. One of the central points of the proof is the identification of the 
particular $\widehat{\gamma}=\widehat{\gamma}(\alpha, \beta)$ which corresponds to a minimum value of $q_{\gamma}$, given $\alpha$ and $\beta$, so that

$$
B_{j, k}^{\alpha}=\max _{\beta \vdash j, \beta \leq \alpha} q_{\alpha}-q_{\beta}-q_{\widehat{\gamma}(\alpha, \beta)} .
$$

What we find, in particular, is that (Lemma 5.11)

$$
\widehat{\gamma}(\alpha, \beta)=\operatorname{srt}(\alpha-\beta),
$$

where srt is the operator that sorts a sequence in nondecreasing order in such a way that the resulting sequence is a partition. So, for instance, if $\alpha=(7,6,2,1)$ and $\beta=(5,2,2)$, we have

$$
\alpha-\beta=(2,4,0,1), \quad \widehat{\gamma}=\operatorname{srt}(\alpha-\beta)=(4,2,1) .
$$

At this point one could reasonably hope in some monotonicity property of the $B_{j, k}^{\alpha}$ with respect to $\alpha$. There is a partial order " $\unlhd$ " in the set of all partitions of $n$, called dominance (see Sect. 5), which plays a crucial role in the representation theory of the symmetric group. It would be nice to prove something like

$$
\alpha \unlhd \alpha^{\prime} \quad \Longrightarrow \quad \lambda_{\max }\left[\alpha, W\left(K_{j, k}\right)\right] \leq \lambda_{\max }\left[\alpha^{\prime}, W\left(K_{j, k}\right)\right] .
$$

Since any nontrivial partition $\alpha$ of $n$ is dominated by the partition $(n-1,1)$, property (4.5), if true, would imply Theorem 3.1 for $\mathscr{G}=K_{j, k}$. Implication (4.5) is unfortunately false. ${ }^{8}$ Nevertheless, our actual strategy is a slight detour from this monotonicity idea. We consider a modified version of the quantities (4.4),

$$
\bar{B}_{j, k}^{\alpha}:=\max _{\beta \vdash j, \beta \leq \alpha} q_{\alpha}-q_{\beta}-q_{\alpha-\beta} .
$$

Then we realize (Proposition 5.10) that $q_{\alpha-\beta} \leq q_{\mathrm{srt}(\alpha-\beta)}$, and thus, by consequence, $B_{j, k}^{\alpha} \leq \bar{B}_{j, k}^{\alpha}$. Using (4.1), one finds (Proposition 5.8) a very simple expression for the quantity $q_{\alpha}-q_{\beta}-q_{\alpha-\beta}$, namely

$$
q_{\alpha}-q_{\beta}-q_{\alpha-\beta}=\beta \cdot(\alpha-\beta)=\sum_{i=1}^{\infty} \beta_{i}\left(\alpha_{i}-\beta_{i}\right) .
$$

At this point one gets a lucky break. In fact:

(a) The monotonicity property (4.5) holds for the quantities $\bar{B}_{j, k}^{\alpha}$ (Proposition 5.9).

(b) If $\alpha=(n-1,1)$, we find that ${ }^{9} \bar{B}_{j, k}^{(n-1,1)}=B_{j, k}^{(n-1,1)}$ (Proposition 5.5).

Combining these facts, we obtain, for any $\alpha \vdash n$ with $\alpha \neq(n)$,

$$
B_{j, k}^{\alpha} \leq \bar{B}_{j, k}^{\alpha} \leq \bar{B}_{j, k}^{(n-1,1)}=B_{j, k}^{(n-1,1)},
$$

and Theorem 3.1 is proven.

\footnotetext{
${ }^{8} \mathrm{~A}$ simple counterexample is given by $K_{3,1}$. One easily finds that $\lambda_{\max }\left[(2,1,1), W\left(K_{3,1}\right)\right]=1$ and $\lambda_{\max }\left[(2,2), W\left(K_{3,1}\right)\right]=0$.

${ }^{9}$ Unless $j=k=1$, but this case is trivial.
} 


\section{Proof of Theorem 3.1}

A complete multipartite graph with $n$ vertices is identified, up to a graph isomorphism, by a partition of $n$, so, if $\sigma=\left(\sigma_{1}, \ldots, \sigma_{p}\right) \vdash n$ with $p \geq 2$, we denote the associated complete multipartite graph with $K_{\sigma}=K_{\sigma_{1}, \ldots, \sigma_{p}}$. The set $\{1, \ldots, n\}$ can be written as a disjoint union

$$
\{1, \ldots, n\}=N_{1}^{\sigma} \cup \cdots \cup N_{p}^{\sigma}
$$

of subsets $N_{k}^{\sigma}$ of cardinality $\sigma_{k}$ given by

$$
N_{k}^{\sigma}:=\left\{\sigma_{1}+\cdots+\sigma_{k-1}+1, \ldots, \sigma_{1}+\cdots+\sigma_{k}\right\}
$$

Let $S_{k}^{\sigma}$ be the subgroup of $S_{n}$ which consists of the permutations $\pi$ such that $\pi(i)=i$ for each $i \in\{1, \ldots, n\} \backslash N_{k}^{\sigma}$. The Young subgroup $S_{\sigma}$ is defined as

$$
S_{\sigma}=S_{\left(\sigma_{1}, \ldots, \sigma_{p}\right)}:=S_{1}^{\sigma} \times \cdots \times S_{p}^{\sigma}
$$

In other words, a permutation $\pi$ belongs to $S_{\sigma}$ if and only if

$$
i \in N_{k}^{\sigma} \Longrightarrow \pi(i) \in N_{k}^{\sigma} \text {. }
$$

The subgroup $S_{\sigma}$ is naturally isomorphic to the (exterior) Cartesian product $S_{\sigma_{1}} \times$ $\cdots \times S_{\sigma_{p}}$.

We observe that the complement of $K_{\sigma}$ is a disjoint union of complete graphs

$$
\bar{K}_{\sigma}=K_{\sigma_{1}} \cup \cdots \cup K_{\sigma_{p}},
$$

and hence

$$
W\left[K_{\sigma}\right]=W\left[K_{n}\right]-W\left[\bigcup_{k=1}^{p} K_{\sigma_{k}}\right],
$$

and thanks to Proposition 4.1, we get, for any irreducible representation $[\alpha]$ of $S_{n}$, the identity ${ }^{10}$

$$
\mathbf{T}^{\alpha}\left[W\left(K_{\sigma}\right)\right]=q_{\alpha} I_{f_{\alpha}}-\mathbf{T}^{\alpha}\left[W\left(\bigcup_{k=1}^{p} K_{\sigma_{k}}\right)\right] .
$$

The quantity $W\left(\bigcup_{k=1}^{p} K_{\sigma_{k}}\right)$ belongs to the group algebra of the Young subgroup $S_{\sigma}$. The irreducible representation $[\alpha]$ of $S_{n}$ is no longer irreducible when restricted to $S_{\sigma}$. The irreducible representations of $S_{\sigma} \cong S_{\sigma_{1}} \times \cdots \times S_{\sigma_{p}}$ are in fact the (outer) tensor products of the irreducible representations of each $S_{\sigma_{i}}$

$$
\operatorname{Irr}\left(S_{\sigma}\right)=\left\{\left[\beta^{1}\right] \otimes \cdots \otimes\left[\beta^{p}\right]: \beta^{i} \vdash \sigma_{i} \text { for each } i=1, \ldots, p\right\}
$$

\footnotetext{
${ }^{10}$ Even though $\alpha$ and $\sigma$ are both partitions of $n$, they play a very different role. $[\alpha]$ is an equivalence class of irreducible representations of $S_{n}$, while $\sigma$ determines the structure of the graph $K_{\sigma}$.
} 
The obvious step at this point is to take advantage of the decomposition

$$
[\alpha] \downarrow_{S_{\sigma}}^{S_{n}}=\bigoplus_{\beta^{1} \vdash \sigma_{1}, \ldots, \beta^{p} \vdash \sigma_{p}} c_{\beta^{1}, \ldots, \beta^{p}}^{\alpha}\left[\beta^{1}\right] \otimes \cdots \otimes\left[\beta^{p}\right]
$$

into a sum of irreducible representations of $S_{\sigma}$. Identities (5.3) and (5.4) and the fact that $W\left(\bigcup_{k=1}^{p} K_{\sigma_{k}}\right) \in \mathbb{C} S_{\sigma}$, imply that the eigenvalues of $\mathbf{T}^{\alpha}[W(\mathscr{G})]$ are of the form $q_{\alpha}-\lambda$, where $\lambda$ is an eigenvalue of

$$
\bigotimes_{k=1}^{p} \mathbf{T}^{\beta_{k}}\left[W\left(\bigcup_{k=1}^{p} K_{\sigma_{k}}\right)\right]
$$

and where $\left(\beta^{1}, \ldots, \beta^{p}\right)$ is a collection of partitions $\beta^{k} \vdash \sigma_{k}$ such that the (multi) Littlewood-Richardson coefficient $c_{\beta^{1}, \ldots, \beta^{p}}^{\alpha}$ is positive. We give a name to these collections of $\beta^{k}$.

Definition 5.1 Given the partitions $\alpha \vdash n$ and $\sigma=\left(\sigma_{1}, \ldots, \sigma_{p}\right) \vdash n$, we say that the $p$-tuple $\left(\beta^{1}, \ldots, \beta^{p}\right)$ of partitions is $(\alpha, \sigma)$-admissible if

(i) each $\beta^{i}$ is a partition of $\sigma_{i}$,

(ii) $c_{\beta^{1}, \ldots, \beta^{p}}^{\alpha}>0$.

We denote with $\operatorname{Adm}(\alpha, \sigma)$ the set of all $(\alpha, \sigma)$-admissible $p$-tuples of partitions.

The spectrum of the matrix in (5.5) can be expressed in a simple form thanks to the fact that $\bigcup_{i=1}^{p} K_{\sigma_{i}}$ is a disjoint union.

Proposition 5.2 Let $\mathscr{H}$ be a finite graph which is the (disjoint) union of subgraphs $\mathscr{H}_{1}, \ldots, \mathscr{H}_{p}$, and let $\sigma_{i}$ be the number of vertices of $\mathscr{H}_{i}$. For each $i=1, \ldots, p$, let $\mathbf{Y}^{i}$ be a representation of $S_{\sigma_{i}}$, and let $\mathbf{Y}$ be the representation of $S_{\sigma}$ given by

$$
\mathbf{Y}:=\bigotimes_{i=1}^{p} \mathbf{Y}^{i}
$$

Then

$$
\operatorname{spec} \mathbf{Y}[W(\mathscr{H})]=\left\{\lambda_{1}+\cdots+\lambda_{p}: \lambda_{i} \in \operatorname{spec} \mathbf{Y}^{i}\left[W\left(\mathscr{H}_{i}\right)\right]\right\}
$$

Proof We have

$$
\begin{aligned}
W(\mathscr{H}) & =\sum_{e \in E(\mathscr{H})} e=\sum_{i=1}^{p} \sum_{e \in E\left(\mathscr{H}_{i}\right)} e=\sum_{i=1}^{p} W\left(\mathscr{H}_{i}\right) \\
& =\sum_{i=1}^{p} 1_{S_{\sigma_{1}}} \cdot \ldots \cdot 1_{S_{\sigma_{i-1}}} \cdot W\left(\mathscr{H}_{i}\right) \cdot 1_{S_{\sigma_{i+1}}} \cdot \ldots \cdot 1_{S_{\sigma_{p}}},
\end{aligned}
$$


where $1_{G}$ stands for the unit element of the group $G$ and of the group algebra $\mathbb{C} G$. If $d_{i}$ is the dimension of the representation $\mathbf{Y}^{i}$ (which will not be confused, hopefully, with the degree of the vertex $i$ ), by the definition of $\mathbf{Y}$ we obtain

$$
\mathbf{Y}[W(\mathscr{H})]=\sum_{i=1}^{p} I_{d_{1}} \otimes \cdots \otimes I_{d_{i-1}} \otimes \mathbf{Y}^{i}\left[W\left(\mathscr{H}_{i}\right)\right] \otimes I_{d_{i+1}} \otimes \cdots \otimes I_{d_{p}} .
$$

Equality (5.6) now follows from a (presumably) standard argument: since $e$ is a transposition, $e^{-1}=e$. We can assume that representations $\mathbf{Y}^{i}$ are unitary, which implies that $\mathbf{Y}^{i}(e)$ is a Hermitian matrix, and thus $\mathbf{Y}^{i}\left[W\left(\mathscr{H}_{i}\right)\right]$ is also Hermitian. ${ }^{11}$ For each $i=1, \ldots, p$, let $\left(u_{j}^{(i)}\right)_{j=1}^{d_{i}}$ be a basis of $\mathbb{C}^{d_{i}}$ consisting of eigenvectors of $\mathbf{Y}^{i}\left[W\left(\mathscr{H}_{i}\right)\right]$. The set of all vectors of the form

$$
u_{j_{1}}^{(1)} \otimes \cdots \otimes u_{j_{p}}^{(p)}
$$

is a basis of $\mathbb{C} \prod_{i} d_{i}$ which consists of eigenvectors of $\mathbf{Y}[W(\mathscr{H})]$. Hence the eigenvalues of $\mathbf{Y}[W(\mathscr{H})]$ are given (5.6).

Thanks to identities (5.3) and (5.4) and to Proposition 5.2 and Proposition 4.1 applied to each $K_{\sigma_{i}}$, we have obtained the following fairly explicit representation for the eigenvalues of $\mathbf{T}^{\alpha}\left[W\left(K_{\sigma}\right)\right]$.

Theorem 5.3 Let $K_{\sigma}$ be the complete multipartite graph associated with the partition $\sigma=\left(\sigma_{1}, \ldots, \sigma_{p}\right)$ of $n$, and let $\mathbf{T}^{\alpha}$ be one of the (equivalent) irreducible representations of $S_{n}$ corresponding to $\alpha=\left(\alpha_{1}, \ldots, \alpha_{r}\right) \vdash n$. Then

$$
\operatorname{spec} \mathbf{T}^{\alpha}\left[W\left(K_{\sigma}\right)\right]=\left\{q_{\alpha}-\sum_{i=1}^{p} q_{\beta^{i}}:\left(\beta_{i}\right)_{i=1}^{p} \text { is }(\alpha, \sigma) \text {-admissible }\right\} .
$$

We now define, for arbitrary weak compositions $\beta^{1}, \ldots, \beta^{p}$, the quantities

$$
\begin{aligned}
b_{\beta^{1}, \ldots, \beta^{p}}^{\alpha} & :=q_{\alpha}-\sum_{i=1}^{p} q_{\beta^{i}}, \\
B_{\sigma}^{\alpha} & :=\max _{\left(\beta^{1}, \ldots, \beta^{p}\right) \in \operatorname{Adm}(\alpha, \sigma)} b_{\beta^{1}, \ldots, \beta^{p}}^{\alpha} .
\end{aligned}
$$

It follows from Theorem 5.3 that

$$
\lambda_{\max }\left[\alpha, W\left(K_{\sigma}\right)\right]=B_{\sigma}^{\alpha} .
$$

In order to prove Theorem 3.1 we must show that

$$
\alpha \neq(n) \quad \Longrightarrow \quad B_{\sigma}^{\alpha} \leq B_{\sigma}^{(n-1,1)} .
$$

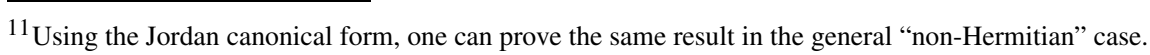


Our plan at this point is the following: we are going to replace the class Adm with a different class Adm* such that

(a) the corresponding maximum

$$
\bar{B}_{\sigma}^{\alpha}:=\max _{\left(\beta^{1}, \ldots, \beta^{p}\right) \in \operatorname{Adm}^{*}(\alpha, \sigma)} b_{\beta^{1}, \ldots, \beta^{p}}^{\alpha}
$$

is easier to evaluate, and, by consequence, we will be able to show that

(b) $\bar{B}_{\sigma}^{\alpha}$ has a useful monotonicity property with respect to the dominance partial order of partitions. A consequence of this monotonicity is that implication (5.13) holds for the quantities $\bar{B}$,

(c) there is a simple and useful relationship between the "true" quantities $B_{\sigma}^{\alpha}$ and their "fake" relatives $\bar{B}_{\sigma}^{\alpha}$, namely: $B_{\sigma}^{\alpha} \leq \bar{B}_{\sigma}^{\alpha}$ and $B_{\sigma}^{\alpha}=\bar{B}_{\sigma}^{\alpha}$ if $\alpha=(n-1,1)$.

From (b) and (c) it follows that (5.13) holds, and hence Theorem 3.1 is proven.

We start then to describe this alternate class Adm*.

Definition 5.4 Given two partitions $\alpha, \sigma$ of $n$, with $\sigma$ of length $p$, we denote with $\operatorname{Adm}^{*}(\alpha, \sigma)$ the set of all $p$-tuples of weak compositions $\left(\gamma^{1}, \ldots, \gamma^{p}\right)$ such that

(i) $\gamma^{i}$ is a weak composition of $\sigma_{i}$,

(ii) $\gamma^{1}+\cdots+\gamma^{p}=\alpha$.

Proposition 5.5 Let $\alpha \vdash n, \sigma=\left(\sigma_{1}, \ldots, \sigma_{p}\right) \vdash n$.

(1) For each $\left(\beta^{i}\right)_{i=1}^{p} \in \operatorname{Adm}(\alpha, \sigma)$, there exists $\left(\gamma^{i}\right)_{i=1}^{p} \in \operatorname{Adm}^{*}(\alpha, \sigma)$ such that

$$
q_{\gamma^{1}}+\cdots+q_{\gamma^{p}} \leq q_{\beta^{1}}+\cdots+q_{\beta^{p}}
$$

By consequence, we have $B_{\sigma}^{\alpha} \leq \bar{B}_{\sigma}^{\alpha}$.

(2) If $\alpha=(n-1,1)$ and $\sigma \neq(1,1, \ldots, 1)$, then $B_{\sigma}^{\alpha}=\bar{B}_{\sigma}^{\alpha}$.

Proof of (1) The crucial point is the following result that we prove at the end of this section.

Proposition 5.6 Let $n=j+k$ with $j, k$ positive integers, and let $\alpha \vdash n, \beta \vdash j, \beta^{\prime} \vdash k$ be such that the Littlewood-Richardson coefficient $c_{\beta, \beta^{\prime}}^{\alpha}$ is positive. Then $\beta \leq \alpha$ and

$$
q_{\beta^{\prime}} \geq q_{\alpha-\beta}
$$

Given Proposition 5.6, we can prove part (1) of Proposition 5.5 by induction on $p \geq 2$. If $p=2$, let $\sigma=(j, k)$ with $j+k=n$, let $\left(\beta, \beta^{\prime}\right) \in \operatorname{Adm}(\alpha, \sigma)$, and define

$$
\left(\gamma, \gamma^{\prime}\right):=(\beta, \alpha-\beta)
$$

The pair $\left(\gamma, \gamma^{\prime}\right)$ clearly belongs to $\operatorname{Adm}^{*}(\alpha,(j, k))$, and hence (5.15) holds thanks to (5.16). 
The general case $p \geq 2$ can be proven by induction on $p$. Assume then that Proposition 5.5 holds for $p-1$ and let $\sigma=\left(\sigma_{1}, \ldots, \sigma_{p}\right) \vdash n$. Define the partitions

$$
\begin{aligned}
\zeta & =\left(\zeta_{1}, \zeta_{2}\right):=\left(\sigma_{1}+\cdots+\sigma_{p-1}, \sigma_{p}\right) \vdash n, \\
\sigma^{\prime} & :=\left(\sigma_{1}, \ldots, \sigma_{p-1}\right) \vdash \zeta_{1} .
\end{aligned}
$$

Since $S_{\sigma}$ is a subgroup of $S_{\zeta}$, we have

$$
[\alpha] \downarrow_{S_{\sigma}}^{S_{n}}=\left([\alpha] \downarrow_{S_{\zeta}}^{S_{n}}\right) \downarrow_{S_{\sigma}}^{S_{\zeta}} .
$$

Thus, from the decompositions

$$
\begin{aligned}
{[\alpha] \downarrow_{S_{\zeta}}^{S_{n}} } & =\sum_{\left(\delta, \delta^{\prime}\right) \in \operatorname{Adm}(\alpha, \zeta)} c_{\delta, \delta^{\prime}}^{\alpha}[\delta] \otimes\left[\delta^{\prime}\right], \\
{[\delta] \downarrow_{S_{\sigma^{\prime}}}^{S_{\zeta_{1}}} } & =\sum_{\left(\beta^{1}, \ldots, \beta^{p-1}\right) \in \operatorname{Adm}\left(\delta, \sigma^{\prime}\right)} c_{\beta^{1}, \ldots, \beta^{p-1}}^{\delta}\left[\beta^{1}\right] \otimes \cdots \otimes\left[\beta^{p-1}\right]
\end{aligned}
$$

we find

$$
c_{\beta^{1}, \ldots, \beta^{p}}^{\alpha}=\sum_{\delta \vdash \zeta_{1}} c_{\delta, \beta^{p}}^{\alpha} c_{\beta^{1}, \ldots, \beta^{p-1}}^{\delta} .
$$

If $\left(\beta^{1}, \ldots, \beta^{p}\right) \in \operatorname{Adm}(\alpha, \sigma)$, the quantity in (5.17) is positive; thus there exists $\delta \vdash \zeta_{1}$ such that both coefficients in the RHS of (5.17) are positive. Pick one such $\delta$. Since $c_{\beta^{1}, \ldots, \beta^{p-1}}^{\delta}>0$, we have, by induction, that there exist $\left(\gamma^{1}, \ldots, \gamma^{p-1}\right) \in \operatorname{Adm}^{*}\left(\delta, \sigma^{\prime}\right)$ such that

$$
q_{\gamma^{1}}+\cdots+q_{\gamma^{p-1}} \leq q_{\beta^{1}}+\cdots+q_{\beta \beta^{-1}} .
$$

Moreover, $c_{\delta, \beta^{p}}^{\alpha}>0$, and thus, by Proposition 5.6 we have

$$
q_{\beta} p \geq q_{\alpha-\delta}
$$

Let $\gamma^{p}:=\alpha-\delta$. From (5.18), (5.19), and the fact that $\left(\gamma^{1}, \ldots, \gamma^{p-1}\right) \in \operatorname{Adm}^{*}\left(\delta, \sigma^{\prime}\right)$ one can easily conclude that $\left(\gamma^{1}, \ldots, \gamma^{p}\right) \in \operatorname{Adm}^{*}(\alpha, \sigma)$ and that inequality (5.15) holds.

Proof of part (2) of Proposition 5.5 We now show that inequality $B_{\sigma}^{\alpha} \leq \bar{B}_{\sigma}^{\alpha}$ is actually an equality when $\alpha=(n-1,1)$ and $\sigma \neq(1,1, \ldots, 1)$.

A simple application of the Littlewood-Richardson rule yields the decomposition

$$
\begin{aligned}
{[n-1,1] \downarrow_{S_{\sigma}}^{S_{n}}=} & (p-1)\left[\sigma_{1}\right] \otimes \cdots \otimes\left[\sigma_{p}\right] \\
& \oplus \bigoplus_{\substack{i=1, \ldots, p \\
\sigma_{i} \geq 2}}\left[\sigma_{1}\right] \otimes \cdots \otimes\left[\sigma_{i-1}\right] \otimes\left[\sigma_{i}-1,1\right] \otimes\left[\sigma_{i+1}\right] \otimes \cdots \otimes\left[\sigma_{p}\right] .
\end{aligned}
$$


Notice that $[m-1,1]$ has degree $m-1$, while $\left[\sigma_{i}\right]$ is the trivial one-dimensional representation, thus the dimension count is correct in (5.20). From (5.20) we can read the list of all $(\alpha, \sigma)$-admissible collections

$$
\operatorname{Adm}(\alpha, \sigma):=\left\{\Psi_{0}\right\} \cup\left\{\Psi_{i}: 1 \leq i \leq p, \sigma_{i} \geq 2\right\},
$$

where

$$
\begin{aligned}
\Psi_{0} & :=\left(\left(\sigma_{1}\right), \ldots\left(\sigma_{p}\right)\right), \\
\Psi_{i} & :=\left(\left(\sigma_{1}\right), \ldots,\left(\sigma_{i-1}\right),\left(\sigma_{i}-1,1\right),\left(\sigma_{i+1}\right), \ldots,\left(\sigma_{p}\right)\right) .
\end{aligned}
$$

On the other hand, it follows from the definition of $\operatorname{Adm}^{*}(\alpha, \sigma)$ that

$$
\operatorname{Adm}^{*}(\alpha, \sigma):=\left\{\Psi_{i}: 1 \leq i \leq p\right\}
$$

If all the $\sigma_{i}$ 's are greater than 1 , then $\operatorname{Adm}^{*}(\alpha, \sigma)$ is a subset of $\operatorname{Adm}(\alpha, \sigma)$, and thus the conclusion is trivial. If some of the $\sigma_{i}$ 's are equal to 1 , then in principle we have to worry about the corresponding $\Psi_{i}$, which belong to $\operatorname{Adm}^{*}(\alpha, \sigma)$ but not to $\operatorname{Adm}(\alpha, \sigma)$. But one can easily compute

$$
\begin{aligned}
& b_{\Psi_{0}}^{\alpha}=q_{(n-1,1)}-\sum_{j=1}^{p} q_{\sigma_{j}}=|E(\mathscr{G})|-|V(\mathscr{G})|, \\
& b_{\Psi_{i}}^{\alpha}=q_{(n-1,1)}-q_{\left(\sigma_{i}-1,1\right)}-\sum_{j=1, j \neq i}^{p} q_{\sigma_{j}}=|E(\mathscr{G})|-|V(\mathscr{G})|+\sigma_{i} .
\end{aligned}
$$

Hence, since, by hypothesis, $\sigma_{1}=\max _{i} \sigma_{i}>1$, we have

$$
B_{\sigma}^{(n-1,1)}=\bar{B}_{\sigma}^{(n-1,1)}=|E(\mathscr{G})|-|V(\mathscr{G})|+\sigma_{1} .
$$

Remark 5.7 Observe that condition $\sigma \neq(1,1, \ldots, 1)$ is necessary. In fact, if $\sigma:=$ $(1,1 \ldots, 1)$, we obtain

$$
B_{\sigma}^{(n-1,1)}=b_{\Psi_{0}}^{\alpha}=|E(\mathscr{G})|-|V(\mathscr{G})|<|E(\mathscr{G})|-|V(\mathscr{G})|+1=\bar{B}_{\sigma}^{\alpha} .
$$

Thanks to condition $\gamma^{1}+\cdots+\gamma^{p}=\alpha$ in the definition of $\operatorname{Adm}^{*}(\alpha, \sigma)$, the quantity $b_{\gamma^{1}, \ldots, \gamma^{p}}^{\alpha}$ has a simple expression.

Proposition 5.8 If $\left(\gamma^{1}, \ldots, \gamma^{p}\right) \in \operatorname{Adm}^{*}(\alpha, \sigma)$, we have

$$
b_{\gamma^{1}, \ldots, \gamma^{p}}^{\alpha}=\sum_{\substack{i, j=1 \\ i<j}}^{p} \gamma^{i} \cdot \gamma^{j},
$$

where $\gamma^{i} \cdot \gamma^{j}$ denotes the canonical inner product $\gamma^{i} \cdot \gamma^{j}:=\sum_{k=1}^{\infty} \gamma_{k}^{i} \gamma_{k}^{j}$. 
Proof The proof is a straightforward computation. Using (4.1) and the fact that $\gamma^{1}+$ $\cdots+\gamma^{p}=\alpha$, we find

$$
\begin{aligned}
q_{\alpha}-\sum_{i=1}^{p} q_{\gamma^{i}} & =\frac{1}{2} \sum_{k=1}^{\infty}\left[\alpha_{k}^{2}-\alpha_{k}(2 k-1)-\sum_{i=1}^{p}\left[\left(\gamma_{k}^{i}\right)^{2}-\gamma_{k}^{i}(2 k-1)\right]\right] \\
& =\frac{1}{2} \sum_{k=1}^{\infty}\left[\left(\sum_{i=1}^{p} \gamma_{k}^{i}\right)^{2}-\sum_{i=1}^{p}\left(\gamma_{k}^{i}\right)^{2}\right]=\sum_{\substack{i, j=1 \\
i<j}}^{p} \gamma^{i} \cdot \gamma^{j}
\end{aligned}
$$

In Sect. 2 we have defined a "componentwise" partial order $\alpha \leq \beta$ in the set of all finite sequences of integers. We introduce now a weaker partial order $\unlhd$, which, following [12], we call dominance order. ${ }^{12}$ If $\alpha, \beta$ are weak compositions, we say that $\beta$ dominates $\alpha$, and we write $\alpha \unlhd \beta$ if

$$
\sum_{i=1}^{r}\left(\beta_{i}-\alpha_{i}\right) \geq 0 \quad \forall r=1,2, \ldots
$$

If $\alpha$ and $\beta$ are weak compositions of the same integer $n, \beta$ dominates $\alpha$ iff (either $\alpha=\beta$ or) the Young diagram of $\beta$ can be obtained from the Young diagram of $\alpha$ by moving a certain number of boxes from a lower row to a higher row. For instance,
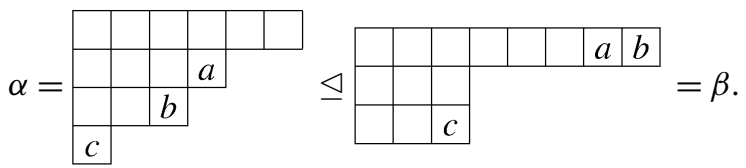

We write $\alpha \sim \beta$ if there exist two integers $j<k$ such that

$$
\alpha_{i}=\beta_{i} \quad \text { for all } i \neq j, k \quad \text { and } \quad \beta_{j}-\alpha_{j}=\alpha_{k}-\beta_{k}=1,
$$

i.e., if $\beta$ is obtained from $\alpha$ by removing one box from the right end of one of its rows and by placing it at the end of a higher row. Then it is obvious that if $\alpha \unlhd \beta$, there exists a finite sequence of "interpolating" weak compositions

$$
\gamma_{1}=\alpha \unlhd \gamma_{2} \unlhd \cdots \unlhd \gamma_{s}=\beta
$$

such that $\gamma_{i} \sim \gamma_{i+1}$. Less obviously, if $\alpha$ and $\beta$ are both partitions of $n$, then the interpolating sequence $\left(\gamma_{i}\right)$ can be chosen in such a way that each $\gamma_{i}$ is also a partition of $n$ (see [12, Theorem 1.4.10], where a slightly different notion of $\sim$ is used; his result implies our statement). The dominance order plays a crucial role in the representation theory of $S_{n}$ and, more generally, in combinatorics. We refer the reader to Sect. 1.4 of [12], where several coimplications of the statement " $\alpha \unlhd \beta$ " are discussed.

We are now ready for part (5) of our "plan" outlined above.

\footnotetext{
${ }^{12}$ One can introduce a third (total) order, namely the lexicographic order, but we do not need it in this paper.
} 
Proposition 5.9 Let $\alpha, \beta$ be two partitions of $n$ with $\alpha \unlhd \beta$.

(1) $q_{\alpha} \leq q_{\beta}$.

(2) $\bar{B}_{\sigma}^{\alpha} \leq \bar{B}_{\sigma}^{\beta}$ for any $\sigma \vdash n$.

End of proof of Theorem 3.1 Before proving Proposition 5.9 we complete the proof of Theorem 3.1. We start with the simple observation that

$$
\begin{aligned}
& \text { If } \alpha \text { is a nontrivial partition of } n \text {, } \\
& \text { i.e., } \alpha \neq(n) \text {, then } \alpha \unlhd(n-1,1) \text {. }
\end{aligned}
$$

If $\sigma=(1,1, \ldots, 1), \mathscr{G}$ is the complete graph $K_{n}$. This case is well known [6]. Anyway, the proof goes as follows: Proposition 4.1 says that $\mathbf{T}^{\alpha}\left[W\left(K_{n}\right)\right]$ has a unique eigenvalue $q_{\alpha}$ of multiplicity equal to the dimension of the representation $\alpha$; in particular, $\lambda_{\max }\left[\alpha, W\left(K_{n}\right)\right]=q_{\alpha}$. Statement (1) of Proposition 5.9 yields $\lambda_{\max }\left[\alpha, W\left(K_{n}\right)\right] \leq \lambda_{\max }\left[(n-1,1), W\left(K_{n}\right)\right]$.

Case $\sigma \neq(1,1, \ldots, 1)$. Thanks to (5.12), (5.27), Proposition 5.5, and Proposition 5.9, we have

$$
\begin{aligned}
\lambda_{\max }\left[\alpha, W\left(K_{\sigma}\right)\right] & =B_{\sigma}^{\alpha} \leq \bar{B}_{\sigma}^{\alpha} \leq \bar{B}_{\sigma}^{(n-1,1)} \\
& =B_{\sigma}^{(n-1,1)}=\lambda_{\max }\left[(n-1,1), W\left(K_{\sigma}\right)\right] .
\end{aligned}
$$

Proof of Proposition 5.9 Thanks to the existence of the interpolating sequence (5.26), we can assume that $\alpha \sim \beta$. Thus there exist two integers $j<k$ such that

$$
\alpha_{i}=\beta_{i} \quad \text { for all } i \neq j, k \quad \text { and } \quad \beta_{j}-\alpha_{j}=\alpha_{k}-\beta_{k}=1 \text {. }
$$

Statement (1) was proven in [6, Lemma 10], and it is a simple computation. In fact, from (4.1), (5.28), and the fact that $\alpha$ is a partition it follows that

$$
q_{\beta}-q_{\alpha}=\left(\alpha_{j}-\alpha_{k}\right)+(k-j+1) \geq k-j+1 \geq 2 .
$$

For the proof of (2), let $\sigma=\left(\sigma_{1}, \ldots, \sigma_{p}\right) \vdash n$. We now show that for each $\left(\gamma^{i}\right)_{i=1}^{p} \in$ $\operatorname{Adm}^{*}(\alpha, \sigma)$, there exists $\left(\delta^{i}\right)_{i=1}^{p} \in \operatorname{Adm}^{*}(\beta, \sigma)$ such that

$$
b_{\gamma^{1}, \ldots, \gamma^{p}}^{\alpha} \leq b_{\delta^{1}, \ldots, \delta^{p}}^{\beta}
$$

Let then $\left(\gamma^{1}, \ldots, \gamma^{p}\right) \in \operatorname{Adm}^{*}(\alpha, \sigma)$ and, for a positive integer $\ell$, which will be determined at the end, define the $p$-tuple $\left(\delta^{i}\right)_{i=1}^{p}$ as follows:

(a) if $i \neq \ell$, we simply let $\delta^{i}=\gamma^{i}$, while

(b) $\delta^{\ell}$ is obtained from $\gamma^{\ell}$ by moving one box from row $k$ to row $j$, i.e.,

$$
\delta_{i}^{\ell}=\gamma_{i}^{\ell} \quad \text { for all } i \neq j, k, \quad \delta_{j}^{\ell}=\gamma_{j}^{\ell}+1, \quad \delta_{k}^{\ell}=\gamma_{k}^{\ell}-1 .
$$


It is clear that $\left(\delta^{i}\right)_{i=1}^{p} \in \operatorname{Adm}^{*}(\beta, \sigma)$ unless $\gamma_{k}^{\ell}=0$, but we will worry about this later. Using Proposition 5.8, we obtain

$$
\begin{aligned}
\Delta_{\ell} b & :=b_{\delta^{1}, \ldots, \delta^{p}}^{\beta}-b_{\gamma^{1}, \ldots, \gamma^{p}}^{\alpha}=\sum_{i, m=1, i<m}^{p}\left[\delta^{i} \cdot \delta^{m}-\gamma^{i} \cdot \gamma^{m}\right] \\
& =\left(\delta^{\ell}-\gamma^{\ell}\right) \cdot \sum_{i=1, i \neq \ell}^{p} \gamma^{i}=\sum_{i=1, i \neq \ell}^{p}\left(\gamma_{j}^{i}-\gamma_{k}^{i}\right) \\
& =\left(\alpha_{j}-\alpha_{k}\right)-\left(\gamma_{j}^{\ell}-\gamma_{k}^{\ell}\right) .
\end{aligned}
$$

From (5.28) it follows that $\alpha_{k} \geq 1$, so the set of "legal" values of $\ell$, that is, those such that $\gamma_{k}^{\ell}>0$, is nonempty. Summing over all these values of $\ell$ and keeping in mind that $\alpha_{j} \geq \alpha_{k}$, we have

$$
\begin{aligned}
\sum_{\ell: \gamma_{k}^{\ell}>0} \Delta_{\ell} b & =\left|\left\{\ell: \gamma_{k}^{\ell}>0\right\}\right|\left(\alpha_{j}-\alpha_{k}\right)-\sum_{\ell: \gamma_{k}^{\ell}>0} \gamma_{j}^{\ell}+\alpha_{k} \\
& \geq \alpha_{j}-\sum_{\ell: \gamma_{k}^{\ell}>0} \gamma_{j}^{\ell}=\sum_{\ell: \gamma_{k}^{\ell}=0} \gamma_{j}^{\ell} \geq 0 .
\end{aligned}
$$

Hence there exists at least one value of $\ell$ such that $\gamma_{k}^{\ell}>0$ and $\Delta_{\ell} b \geq 0$. If we then define $\left(\delta_{i}\right)_{i=1}^{p}$ using this value of $\ell$, we get (5.29).

Proof of Proposition 5.6 Let $n=j+k, \alpha \vdash n, \beta \vdash j, \gamma \vdash k$, and assume that the Littlewood-Richardson coefficient $c_{\beta, \gamma}^{\alpha}$ is positive. The fact that $\beta \leq \alpha$, i.e., that $\beta_{i} \leq \alpha_{i}$ for each $i$, trivially follows from the Littlewood-Richardson rule, which we discuss in Sect. 6. We must prove that $q_{\gamma} \geq q_{\alpha-\beta}$. The difference $\alpha-\beta$ is a weak composition of $k$ but not necessarily a partition. We can nevertheless obtain a partition by sorting the entries of $\alpha-\beta$ in nonincreasing order (and dropping the trailing zeros). We denote this partition with $\operatorname{srt}(\alpha-\beta)$. So, if

$$
\alpha-\beta=\delta=\left(\delta_{1}, \ldots, \delta_{r}\right)
$$

we have

$$
\operatorname{srt}(\delta)=\left(\delta_{\pi(1)}, \ldots, \delta_{\pi(r)}\right) \quad \text { with } \delta_{\pi(1)} \geq \cdots \geq \delta_{\pi(r)},
$$

where $\pi$ is a suitable permutation in $S_{r}$. We start by observing that this sorting procedure does not decrease the quantity $q$.

Proposition 5.10 Let $\alpha, \beta$ be two weak compositions of $n$. Assume that $\beta$ is obtained from $\alpha$ by switching two elements $\alpha_{j}, \alpha_{k}$ with $j<k$, i.e.,

$$
\beta=\left(\alpha_{1}, \ldots, \alpha_{j-1}, \alpha_{k}, \alpha_{j+1}, \ldots, \alpha_{k-1}, \alpha_{j}, \alpha_{k+1}, \ldots\right)
$$


Then $^{13} \alpha_{j}<\alpha_{k}$ if and only if $q_{\alpha}<q_{\beta}$. By consequence, $q_{\mathrm{srt}(\alpha)} \geq q_{\alpha}$ for any weak composition $\alpha$.

Proof From (4.1) we get

$$
\begin{aligned}
q_{\beta}-q_{\alpha}= & \frac{1}{2}\left[\alpha_{k}\left(\alpha_{k}-2 j+1\right)-\alpha_{j}\left(\alpha_{j}-2 j+1\right)\right. \\
& \left.+\alpha_{j}\left(\alpha_{j}-2 k+1\right)-\alpha_{k}\left(\alpha_{k}-2 k+1\right)\right] \\
= & \left(\alpha_{j}-\alpha_{k}\right)(j-k) .
\end{aligned}
$$

The proposition follows, since $j<k$.

The central ingredient of the proof of Proposition 5.6 is the following property of the Richardson-Littlewood coefficients, which we prove in Sect. 6 and which identifies the partition $\gamma$ with "minimal content."

Lemma 5.11 Let $\alpha \vdash n$ and $\beta \vdash j$ with $j<n$ and $\beta \leq \alpha$. Let $\widehat{\gamma}:=\operatorname{srt}(\alpha-\beta)$, so that $\widehat{\gamma}$ is a partition of $k=n-j$.

(1) The Littlewood-Richardson coefficient $c_{\beta, \widehat{\gamma}}^{\alpha}$ is positive.

(2) If $\gamma$ is a partition of $k$ such that $c_{\beta, \gamma}^{\alpha}>0$, then $\gamma \unrhd \widehat{\gamma}$.

Given Lemma 5.11, the proof of Proposition 5.6 readily follows. In fact, from $\gamma \unrhd \widehat{\gamma}$, statement (1) of Proposition 5.9, and Proposition 5.10 we obtain

$$
q_{\gamma} \geq q_{\widehat{\gamma}}=q_{\mathrm{srt}(\alpha-\beta)} \geq q_{\alpha-\beta} .
$$

Remark 5.12 Theorem 3.1 states that, if $\mathscr{G}$ is complete multipartite, then

$$
\alpha \unlhd(n-1,1) \quad \Longrightarrow \quad \lambda_{\max }[\alpha, W(\mathscr{G})] \leq \lambda_{\max }[(n-1,1), W(\mathscr{G})]
$$

One may wonder whether the following "strict version" of this implication holds:

$$
\alpha \triangleleft(n-1,1) \quad \Longrightarrow \quad \lambda_{\max }[\alpha, W(\mathscr{G})]<\lambda_{\max }[(n-1,1), W(\mathscr{G})]
$$

This would imply that the first nontrivial eigenvalues of $\Delta \mathscr{G}$ and $\Delta_{\text {Cay }(\mathscr{G})}$ are not only equal but also have the same multiplicity. But (5.33) is false. Take, in fact, $K_{2,2}$. It is easy to verify that

$$
\lambda_{\max }\left((2,2), W\left[K_{2,2}\right]\right)=\lambda_{\max }\left((3,1), W\left[K_{2,2}\right]\right)=2 .
$$

\footnotetext{
${ }^{13}$ Recall that we have defined $q_{\alpha}$ for weak compositions by formula (4.1).
} 


\section{Littlewood-Richardson tableaux with minimal content}

\subsection{The Littlewood-Richardson rule}

Since we are going to deal with the mechanisms of the Littlewood-Richardson rule, it might be a good idea to briefly describe how it works. We need to define skew tableaux, semistandard tableaux, lattice permutations and content of a tableau. Impatient readers who are not acquainted with this rule and who prefer worked examples to abstract definitions might try staring at Table 2 for a couple of minutes. ${ }^{14}$

Let as usual $j, k$ be two positive integers with $n=j+k$. Given $\alpha \vdash n$ and $\beta \vdash j$ with $\beta \leq \alpha$, the skew diagram of shape $\alpha / \beta$ is the set of boxes obtained by erasing in the Young diagram $\alpha$ all boxes which also appear in $\beta$. Let, for example, $\alpha=$ $(7,6,3,1)$ and $\beta=(5,2,1)$. Let us draw $\alpha$ and cross all boxes which belong to $\beta$. The skew diagram of shape $\alpha / \beta$ is the set of all uncrossed boxes

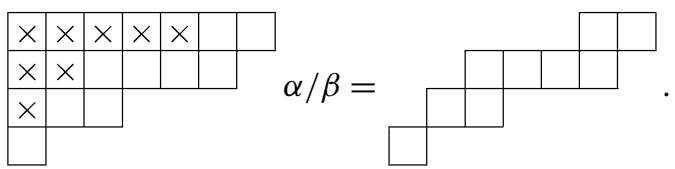

A skew tableau is a skew diagram with a positive integer placed in each box. A skew tableau is called semistandard if each row is nondecreasing and each column is strictly increasing. A lattice permutation is a finite sequence of positive integers $\omega=\left(\omega_{1}, \omega_{2}, \ldots, \omega_{s}\right)$ such that for each $k=1, \ldots, s$, the number of times that any given integer $i$ appears in the initial subsequence $\left(\omega_{1}, \ldots, \omega_{k}\right)$ cannot exceed the number of times that $i-1$ appears in the same subsequence. In other words,

$$
\left|\left\{j \leq k: \omega_{j}=a\right\}\right| \geq\left|\left\{j \leq k: \omega_{j}=b\right\}\right| \quad \forall k=1, \ldots, s \forall a<b .
$$

For instance, if we let

$$
\omega:=(1,1,2,1,3,2,2,3,1,1,2), \quad \omega^{\prime}:=(1,1,2,1,3,2,3, \underline{3}, 1,1,2),
$$

then $\omega$ is a lattice permutation, but $\omega^{\prime}$ is not, since, when we arrive at the underlined digit $\underline{3}$, we realize that we have encountered along the way more 3's than 2's.

The content of a finite sequence of positive integers $\omega=\left(\omega_{1}, \ldots, \omega_{s}\right)$ is the sequence $\gamma=\left(\gamma_{1}, \gamma_{2}, \ldots\right)$, where $\gamma_{i}$ is the number of times the integer $i$ appears in $\omega$. The content of a (skew) Young tableau $t$ is the content of the sequence of all the integers which appear in $t$, listed in any (obviously arbitrary) order. So, for instance,

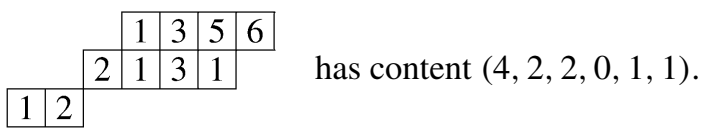

\footnotetext{
${ }^{14}$ It did not work for the author though!
} 
Table 2 LR tableaux of shape $\alpha / \beta$ and content $\gamma$

\begin{tabular}{lcll}
\hline$\alpha=(6,5,3,1) \beta=(5,2,1)$ & \\
\hline LR tableau & $\gamma$ & LR tableau & $\gamma$ \\
\hline
\end{tabular}

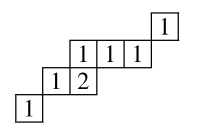

$(6,1)$
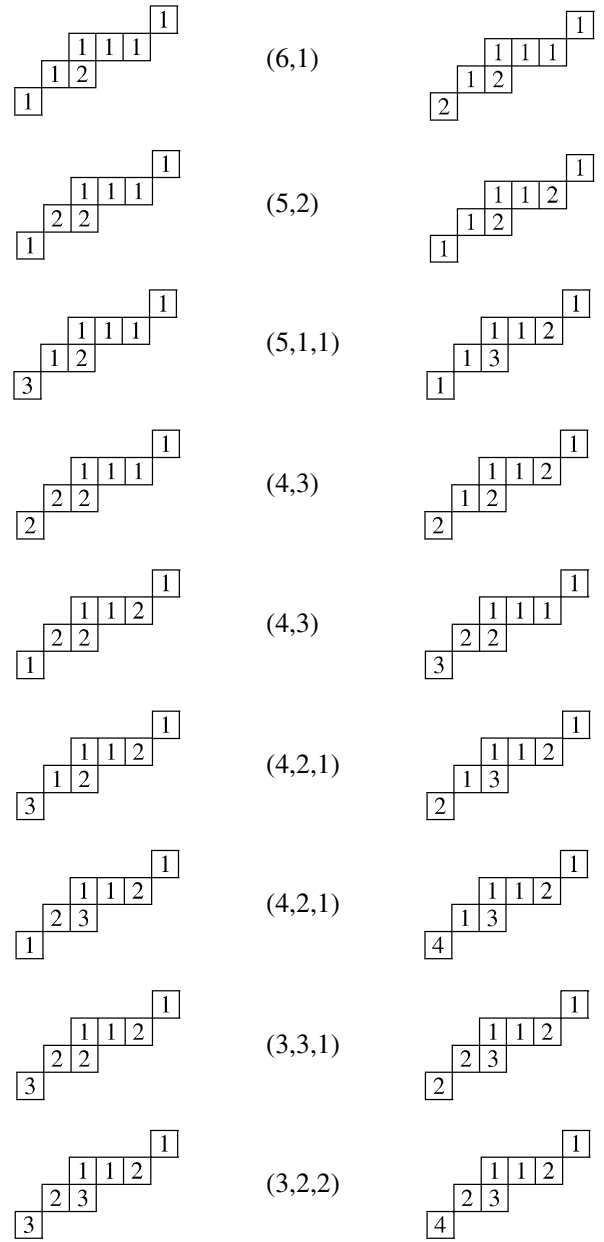
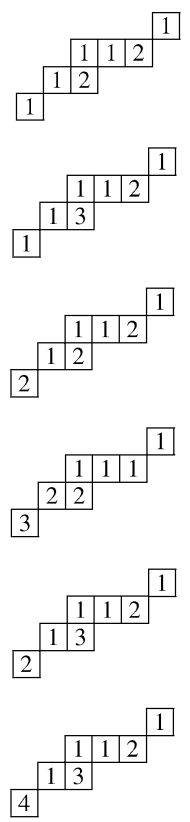

We are finally able to state the Littlewood-Richardson rule. For a proof, see, for instance, [12] or [15].

Littlewood-Richardson rule The coefficient $c_{\beta, \gamma}^{\alpha}$ which appears in (4.3) is equal to the number of semistandard skew tableaux of shape $\alpha / \beta$ and content $\gamma$, which yield lattice permutations when we read their entries from right to left and downward. We will call these tableaux LR tableaux.

Table 2 shows all LR tableaux of shape $\alpha / \beta$ with content $\gamma$ for $\alpha=(6,5,3,1)$ and $\beta=(5,2,1)$. Notice that the minimal $\gamma$ is the last entry of the table, $\gamma=(3,2,1,1)=$ $\operatorname{srt}(\alpha-\beta)$, in accord with Lemma 5.11. 


\subsection{Proof of Lemma 5.11}

Let $t$ be an LR tableau of shape $\alpha / \beta$, and let $\delta=\alpha-\beta$. Since $\beta \leq \alpha$, in general, $\delta$ is a weak composition of $k:=|\alpha|-|\beta|$, so it can have (nontrailing) zeros. In the next remark we get rid of these zeros, reducing to the case $\delta \models k$. This is really irrelevant, as we will see, but it simplifies some statements. For this purpose, we introduce the notation

$$
\beta \ll \alpha \stackrel{\text { def }}{\Longleftrightarrow} \beta_{i}<\alpha_{i} \quad \text { for all } i \text { such that } \alpha_{i}>0,
$$

so that $\beta \ll \alpha$ implies that $\alpha-\beta$ is a composition.

Remark 6.1 (Reduction to the case $\beta \ll \alpha$ ) Assume that the Young diagrams of $\alpha$ and $\beta$ have some corresponding rows of equal length $\alpha_{i}=\beta_{i}$. Let $\bar{\alpha}$ and $\bar{\beta}$ be the Young diagrams obtained by eliminating in $\alpha$ and $\beta$ all corresponding rows of equal length. Then it is obvious that the number of LR tableaux of shape $\alpha / \beta$ and content $\gamma$ is not affected by the simultaneous replacements of $\alpha$ with $\bar{\alpha}$ and $\beta$ with $\bar{\beta}$. Since, on the other hand, $\operatorname{srt}(\alpha-\beta)=\operatorname{srt}(\bar{\alpha}-\bar{\beta})$, it follows that if Lemma 5.11 holds when $\beta \ll \alpha$, it also holds for $\beta \leq \alpha$.

It is our intention to concatenate the rows of an LR tableau $t$ into a single sequence of positive integers, of length $|\delta|$, that we denote by $\vartheta(t)$. Rather than keep struggling with our primordial instinct to read (and think) from left to right, ${ }^{15}$ we first flip each row of $t$ and then concatenate the rows from top to bottom. So, for instance, if

$$
\alpha=(7,6,4,3), \quad \beta=(4,2,1,1), \quad \gamma=(6,3,2,1), \quad \delta=(3,4,3,2),
$$

an example of an LR tableaux $t$ of shape $\alpha / \beta$, content $\gamma$, and relative sequence $\vartheta(t)$ is

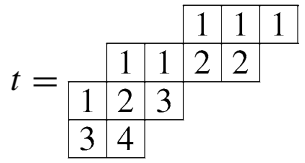

$$
\begin{aligned}
& \vartheta(t)=(\underbrace{1,1,1}_{N_{1}^{\delta}}, \underbrace{2,2,1,1}_{N_{2}^{\delta}}, \underbrace{3,2,1}_{N_{3}^{\delta}}, \underbrace{4,3}_{N_{4}^{\delta}}) .
\end{aligned}
$$

We observe that $\vartheta(t)$ is nonincreasing in each subinterval corresponding a row of $t$.

Definition 6.2 Given $\delta=\left(\delta_{1}, \ldots, \delta_{r}\right) \models n$, we say that a sequence of $n$ positive integers is $\delta$-nonincreasing if it is nonincreasing in each subinterval $N_{i}^{\delta}$ (recall definition (5.1)). For future purposes, it is convenient to stretch a little bit (in an obvious way) this definition, in order to accommodate also sequences shorter than $|\delta|$. If $m<n=|\delta|$, a sequence of $m$ positive integers is called $\delta$-nonincreasing if the sequence of length $n$ obtained by adding $n-m 1$ 's at the end is $\delta$-nonincreasing.

${ }^{15}$ We apologize to native right-to-left thinkers. 
We denote with $\Omega_{\delta}$ the set of all sequences $\omega=\left(\omega_{1}, \ldots, \omega_{|\delta|}\right)$ of positive integers such that

(i) $\omega$ is a lattice permutation,

(ii) $\omega$ is $\delta$-nonincreasing.

We have then:

Proposition 6.3 Given $\alpha \vdash n$ and $\beta \vdash j$ with $\beta \ll \alpha$, the mapping $t \mapsto \vartheta(t)$ is an injection from the set of all LR tableaux of shape $\alpha / \beta$ into $\Omega_{\alpha-\beta}$.

Proof It is obvious from the construction of $\vartheta(t)$ and from the definition of LR tableau.

Remark 6.4 Notice that in general, given $\alpha$ and $\beta$, and letting $\delta:=\alpha-\beta$, the mapping

$$
\vartheta:\{\text { LR tableaux of shape } \alpha / \beta\} \mapsto \Omega_{\delta}
$$

is not surjective. There will be in fact elements $\omega \in \Omega_{\delta}$ that, when "disassembled back and flipped" in order to restore a tableau, are going to produce a tableau $t$ which fails to satisfy the requirement of having increasing columns. In particular, $\omega=(1,1, \ldots, 1)$ is always an element of $\Omega_{\delta}$, but it is not in the image of $\vartheta$ unless $\beta_{i} \geq \alpha_{i+1}$ for all $i$, which means that the rows of skew tableaux of shape $\alpha / \beta$ are shifted in such a way that they do not "overlap," like, for instance,

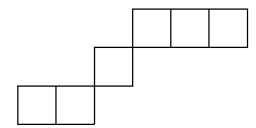

So, given $\alpha$ and $\beta$, the set $\Omega_{\delta}$ contains "good" sequences as well as "extraneous" sequences which do not correspond to any LR tableau of shape $\alpha / \beta$. On the other hand, the advantage of dealing with $\Omega_{\delta}$ is that this set depends on the pair $(\alpha, \beta)$ only through their difference $\alpha-\beta$. In the end, this works because, quite remarkably, the solution to the problem of finding the minimal $\gamma$ such that $c_{\beta, \gamma}^{\alpha}>0$ does indeed depend only on $\alpha-\beta$ !

We now describe a simple algorithm for constructing, for a given $\delta \models k$, a particular sequence $\widehat{\omega} \in \Omega_{\delta}$ which has the property of having minimal content with respect to all other $\omega \in \Omega_{\delta}$. Then we show that the content of $\widehat{\omega}$ is $\operatorname{srt}(\delta)$. Finally, we prove that $\widehat{\omega}$ is a good sequence, meaning that there exists an LR tableau $t$ such that $\widehat{\omega}=\vartheta(t)$, completing in this way the proof of Lemma 5.11.

Let then $\delta$ be a composition of $k$. The sequence $\widehat{\omega}$ in $\Omega_{\delta}$ is defined recursively as follows:

(a) start with $\widehat{\omega}_{1}=1$ (mandatory),

(b) given $\left(\widehat{\omega}_{1}, \ldots, \widehat{\omega}_{i-1}\right)$, choose $\widehat{\omega}_{i}$ as the largest integer such that the sequence $\left(\widehat{\omega}_{1}, \ldots, \widehat{\omega}_{i}\right)$ satisfies (i) and (ii) in the definition of $\Omega_{\delta}$. 
We call $\widehat{\omega}$ the minimal sequence in $\Omega_{\delta} .{ }^{16}$ We observe that this algorithm can never get stuck. Let, in fact, $X_{i}$ be the set of possible choices for $\widehat{\omega}_{i}$, i.e., the set of integers $s$ such that $\left(\widehat{\omega}_{1}, \ldots, \widehat{\omega}_{i-1}, s\right)$ satisfies (i) and (ii) in the definition of $\Omega_{\delta}$. Then $X_{i}$ is nonempty ( 1 is always a possible choice) and bounded (a trivial bound for being a lattice permutation is $\left.s=\widehat{\omega}_{i} \leq i\right)$, thus the maximum of $X_{i}$ exists.

For example, if $\delta=(3,4,2,4)$, the minimal sequence is given by

$$
\widehat{\omega}=(\underbrace{1,1,1}_{N_{1}^{\delta}}, \underbrace{2,2,2,1}_{N_{2}^{\delta}}, \underbrace{3,3}_{N_{3}^{\delta}}, \underbrace{4,4,3,2}_{N_{4}^{\delta}}) .
$$

Notice that, at least in this case, the content of $\widehat{\omega}$ is $(4,4,3,2)=\operatorname{srt}(\delta)$.

Proposition 6.5 Let $\widehat{\omega}$ be the minimal sequence in $\Omega_{\delta}$.

(1) If $z_{\ell}$ is the first integer of the subinterval $N_{\ell}^{\delta}$, we have $\widehat{\omega}_{z_{\ell}}=\ell$.

(2) $\max \left\{\widehat{\omega}_{i}: i \in N_{\ell}^{\delta}\right\}=\ell$.

(3) If $p:=\widehat{\omega}_{i}>\widehat{\omega}_{i+1}=: m$, then $\left|\left\{j \leq i: \widehat{\omega}_{j}=m\right\}\right|=\left|\left\{j \leq i: \widehat{\omega}_{j}=p\right\}\right|$.

Proof Let $z_{\ell}=\delta_{1}+\cdots+\delta_{\ell-1}+1$ be the first integer in $N_{\ell}^{\delta}$. For $\ell=1$, we have $z_{\ell}=1$ and, necessarily, $\widehat{\omega}_{1}=1$. Assume then that $\widehat{\omega}_{z_{j}}=j$ for $j=1, \ldots, \ell-1$. Since $\widehat{\omega}$ is $\delta$-nonincreasing, we have

$$
\max \left\{\widehat{\omega}_{i}: i \in N_{1}^{\delta} \cup \cdots \cup N_{\ell-1}^{\delta}\right\}=\widehat{\omega}_{z \ell-1}=\ell-1 .
$$

Imagine to have constructed $\left(\widehat{\omega}_{1}, \ldots, \widehat{\omega}_{z \ell-1}\right)$. Since $z_{\ell}$ is at the beginning of $N_{\ell}^{\delta}$, for picking $\widehat{\omega}_{z \ell}$, we do not worry about $\delta$-nonincreasing. On the other hand, from (6.5) it follows that each integer from 1 to $\ell-1$ has already appeared in $\widehat{\omega}$ at least once, while no integer greater than or equal to $\ell$ has yet appeared. By consequence, $\widehat{\omega}_{z \ell}=\ell$. This proves part (1) by induction. Statement (2) follows from (1) and from the fact that $\widehat{\omega}$ is $\delta$-nonincreasing.

Assume $p=\widehat{\omega}_{i}>\widehat{\omega}_{i+1}=m$. This means, by the definition of $\widehat{\omega}$, that none of the integers from $m+1$ to $p$ can be picked as $\widehat{\omega}_{i+1}$, since that would violate the lattice permutation property. Hence we must have

$$
\begin{aligned}
\left|\left\{j \leq i: \widehat{\omega}_{j}=m-1\right\}\right| & >\left|\left\{j \leq i: \widehat{\omega}_{j}=m\right\}\right| \\
& =\left|\left\{j \leq i: \widehat{\omega}_{j}=m+1\right\}\right|=\cdots=\left|\left\{j \leq i: \widehat{\omega}_{j}=p\right\}\right| .
\end{aligned}
$$

Proposition 6.6 Let $\omega \in \Omega_{\delta}$ for some $\delta \models n$, and let $\gamma$ be the content of $\omega$. Let also $\widehat{\gamma}$ be the content of the minimal sequence $\widehat{\omega}$. Then

$$
\gamma \unrhd \widehat{\gamma}=\operatorname{srt}(\delta)
$$

\footnotetext{
${ }^{16}$ The reader may rightfully feel that we are testing his patience, by calling minimal a sequence where we pick at each step the largest possible value. But, again, $\widehat{\omega}$ has minimal content. In case someone were wondering, $\widehat{\omega}$ is not maximal in $\Omega_{\delta}$, in general, with respect to the componentwise partial order.
} 
Proof We first observe that, given two arbitrary finite sequences $\omega, \bar{\omega}$ of positive integers with respective contents $\beta$ and $\bar{\beta}$, we have

$$
\omega \leq \bar{\omega} \Longleftrightarrow \beta \unrhd \bar{\beta} .
$$

This simply follows from the definition (5.24) of dominance and from the identity $\sum_{i=1}^{s} \beta_{i}=\left|\left\{j: \omega_{j} \leq s\right\}\right|$.

It is well known $[12,1.4 .11]$ that the dominance order is reversed on conjugate partitions (2.1), that is,

$$
\alpha \unlhd \beta \quad \Longleftrightarrow \quad \alpha^{\prime} \unrhd \beta^{\prime},
$$

and thus we are going to prove that $\gamma^{\prime} \unlhd \widehat{\gamma}^{\prime}$. Given $\omega=\left(\omega_{1}, \ldots, \omega_{n}\right) \in \Omega_{\delta}$, we define the running multiplicity of $\omega$ as the sequence $v=\left(v_{1}, \ldots, v_{n}\right)$, where

$$
v_{i}:=\left|\left\{j \leq i: \omega_{j}=\omega_{i}\right\}\right| .
$$

In other words, $v_{i}$ is the number of times that $\omega_{i}$ has appeared in the sequence "up to that point." Proposition 6.6 is a consequence of the following lemma.

\section{Lemma 6.7}

(A) The content of $v$ is $\gamma^{\prime}$, that is, $\gamma_{i}^{\prime}=\left|\left\{j: v_{j}=i\right\}\right|$. In other words, the content of the running multiplicity of $\omega$ is the dual of the content of $\omega$.

(B) The sequence $v$ is strictly increasing on each subinterval of $\{1, \ldots, n\}$ on which $\omega$ is nonincreasing. In particular, since $\omega$ is $\delta$-nonincreasing, $v$ is strictly increasing on each subinterval $N_{i}^{\delta}$.

(C) If $\widehat{v}$ is the running multiplicity of $\widehat{\omega}$, we have, denoting the length of $\delta$ with $r$,

$$
\widehat{v}=(\underbrace{1,2,3, \ldots, \delta_{1}}_{N_{1}^{\delta}}, \underbrace{1,2,3, \ldots, \delta_{2}}_{N_{2}^{\delta}}, \ldots, \underbrace{1,2,3, \ldots, \delta_{r}}_{N_{r}^{\delta}}) .
$$

(D) $\widehat{\gamma}=\operatorname{srt}(\delta)$.

From (A), (B), (C), and (D) Proposition 6.6 follows. In fact,

$$
\begin{array}{rlrl}
(\mathrm{B})+(\mathrm{C}) & \Longrightarrow \widehat{v} \leq v & \\
& \Longrightarrow \widehat{\gamma}^{\prime} \unrhd \gamma^{\prime} \quad & \\
& \Longrightarrow \gamma \unrhd \widehat{\gamma}=\operatorname{srt}(\delta) & & \text { [thanks to (A) and (6.6)] }
\end{array}
$$

We are then left with the proof Lemma 6.7.

Proof of (A) $\gamma_{i} \geq m$ means that $i$ appears at least $m$ times in $\omega$. Hence there exists a positive integer $k_{i} \leq n$ which marks the $m$ th appearance of $i$ in $\omega$. Clearly $v_{k_{i}}=m$. Vice-versa, if $v_{k}=m$, the integer $\omega_{k}$ appears $m$ times in $\left\{\omega_{1}, \ldots, \omega_{k}\right\}$, thus we have $\gamma_{\omega_{k}} \geq m$. We have then, for fixed $\omega$ and $m$, a bijection

$$
\left\{i: \gamma_{i} \geq m\right\} \ni i \longleftrightarrow k_{i} \in\left\{k: v_{k}=m\right\}
$$


which implies

$$
\gamma_{m}^{\prime}=\left|\left\{i: \gamma_{i} \geq m\right\}\right|=\left|\left\{k: v_{k}=m\right\}\right|,
$$

i.e., that $\gamma^{\prime}$ is the content of $\nu$.

Proof of $(B)$ If $\omega_{i+1} \leq \omega_{i}$, since $\omega$ is a lattice permutation, we have

$$
\begin{aligned}
v_{i+1} & =\left|\left\{j \leq i+1: \omega_{j}=\omega_{i+1}\right\}\right|=\left|\left\{j \leq i: \omega_{j}=\omega_{i+1}\right\}\right|+1 \\
& \geq\left|\left\{j \leq i: \omega_{j}=\omega_{i}\right\}\right|+1=v_{i}+1 .
\end{aligned}
$$

Proof of $(C)$ Let $z_{\ell}$ be the first integer in $N_{\ell}^{\delta}$. Proposition 6.5 implies that the integer $\ell$ appears for the first time in $\widehat{\omega}$ at position $z_{\ell}$, and hence $\widehat{v}_{z \ell}=1$.

In order to prove (6.8) we show that, within each subinterval $N_{\ell}^{\delta}$, we have $\widehat{v}_{i+1}=$ $\widehat{v}_{i}+1$. If $\widehat{\omega}_{i+1}=\widehat{\omega}_{i}$, this is a trivial consequence of the definition of $\widehat{v}$. On the other hand, if $p:=\widehat{\omega}_{i}>\widehat{\omega}_{i+1}=: m$, thanks to Proposition 6.5, we have

$$
\begin{aligned}
\widehat{v}_{i+1} & =\left|\left\{j \leq i+1: \omega_{j}=m\right\}\right|=\left|\left\{j \leq i: \omega_{j}=m\right\}\right|+1 \\
& =\left|\left\{j \leq i: \omega_{j}=p\right\}\right|+1=\widehat{v}_{i}+1 .
\end{aligned}
$$

Proof of $(D)$ Let $\varepsilon:=\operatorname{srt}(\delta)$, so that $\varepsilon$ is a partition of $|\delta|$. From statement (A) applied to $\widehat{v}$, from the explicit expression of $\widehat{v}$ given by (6.8), and from the definition of duality (2.1) it follows that the content of $\widehat{v}$ is given by

$$
\widehat{\gamma}_{i}^{\prime}=\left|\left\{j: \widehat{v}_{j}=i\right\}\right|=\left|\left\{j: \delta_{j} \geq i\right\}\right|=\left|\left\{j: \varepsilon_{j} \geq i\right\}\right|=\varepsilon_{i}^{\prime} .
$$

But $\gamma$ and $\varepsilon$ are both partitions, so we can take the dual and get (D). Lemma 6.7 and Proposition 6.6 are thus proven.

In order to complete the proof of Lemma 5.11, one last step is required, namely to check that the minimal sequence $\widehat{\omega}$ in $\Omega_{\delta}$ corresponds to an actual LR tableaux.

Proposition 6.8 Let $\delta$ be a composition of $k$, and let $\widehat{\omega}$ be the minimal sequence in $\Omega_{\delta}$ with content $\widehat{\gamma}=\operatorname{srt}(\delta)$. If $\alpha$ is a partition of $n$ and $\beta$ is a partition of $n-k$ such that $\alpha-\beta=\delta$, then there exists an LR tableau $t$ of shape $\alpha / \beta$ and content $\widehat{\gamma}$ such that $\vartheta(t)=\widehat{\omega}$.

Proof Let $z_{\ell}$ be the first integer in the interval $N_{\ell}^{\delta}$. Hence,

$$
\widehat{\omega}=(\underbrace{\widehat{\omega}_{z_{1}} \ldots, \widehat{\omega}_{\delta_{1}}}_{N_{1}^{\delta}}, \underbrace{\widehat{\omega}_{z_{2}}, \ldots, \widehat{\omega}_{z_{2}+\delta_{2}-1}}_{N_{2}^{\delta}}, \ldots, \underbrace{\widehat{\omega}_{z_{r}}, \ldots, \widehat{\omega}_{z_{r}+\delta_{r}-1}}_{N_{r}^{\delta}}) .
$$

In order to reconstruct a tableau $t$ from $\widehat{\omega}$, we proceed in three steps.

(s1) We arrange the restrictions of $\widehat{\omega}$ to each subinterval $N_{\ell}^{\delta}$ as the rows of an (improper) tableau with a left aligned border. We denote this tableau with $t^{\#}$.

(s2) For each $\ell=2, \ldots, r$, we shift the $\ell$ th row of $t^{\#}$ by $\alpha_{1}-\alpha_{\ell}$ positions to the right and denote this tableau with $\tilde{t}$. 
(s3) Finally, we flip $\tilde{t}$ horizontally and get the tableau $t$ which is a skew tableau of shape $\alpha / \beta$.

Consider, for example, the sequence $\widehat{\omega}$ in (6.4). If $\alpha=(8,7,5,4)$, transformations (s1), (s2), and (s3) yield

\begin{tabular}{|l|l|l|l|}
\hline 1 & 1 & 1 & \multicolumn{1}{|c|}{} \\
\hline 2 & 2 & 2 & 1 \\
\hline 3 & 3 & \multicolumn{2}{|c}{} \\
\hline 4 & 4 & 3 & 2 \\
\hline \multicolumn{4}{c}{$t^{\#}$}
\end{tabular}
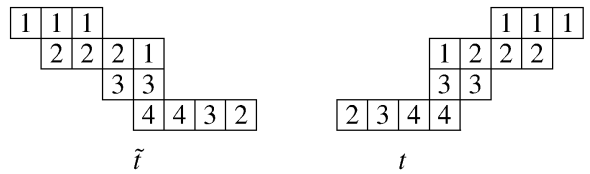

We denote with $t_{i j}^{\#}$ (with $j=1, \ldots, \delta_{i}$ ) the integer contained in the $j$ th box of the $i$ th row of $t^{\#}$ (as if $t^{\#}$ were a matrix). Recall that all we have to check is that the columns of $t$ are strictly increasing, since the other properties of the LR tableaux (nondecreasing rows and the lattice permutation property) are inherited from $\widehat{\omega}$. We are going to prove that:

\section{Lemma 6.9}

(A) The columns of $t^{\#}$ are strictly increasing. ${ }^{17}$

(B) Steps (s2) and (obviously) (s3) described above preserve the property of having strictly increasing columns.

Proof of (A) If $q$ is a positive integer, let

$$
h_{\ell}(q):=\text { number of } q \text { 's appearing in the first } \ell \text { rows of } t^{\#} .
$$

We claim that

$$
\begin{aligned}
& \text { if } q \text { appears in the } \ell \text { th row of } t^{\#} \text {, its first appearance } \\
& \text { occurs at position } h_{\ell-1}(q)+1 \text {, thus } q \text { occupies } \\
& \text { the positions: } h_{\ell-1}(q)+1, h_{\ell-1}(q)+2, \ldots, h_{\ell}(q) \text {. }
\end{aligned}
$$

The second part of the statement trivially follows from the first part and from the fact that $h_{\ell}(q)-h_{\ell-1}(q)$ is the number of $q$ 's in the $\ell$ th row. Let $p_{1}>p_{2}>\cdots>p_{s}$ be the distinct integers which appear in the $\ell$ th row of $t^{\#}$. Thanks to Proposition 6.5, we already know that $p_{1}=\ell$ and that $h_{\ell-1}(\ell)=0$, and hence (6.11) holds for $p_{1}$. Assume now that (6.11) holds for $p_{i}$. If we let $j=h_{\ell}\left(p_{i}\right)$, then $j$ is the last position where $p_{i}$ appears, so

$$
\widehat{\omega}_{z \ell+j-1}=t_{\ell, j}^{\#}=p_{i}>p_{i+1}=t_{\ell, j+1}^{\#}=\widehat{\omega}_{z \ell+j} .
$$

Statement (3) of Proposition 6.5 implies that $h_{\ell}\left(p_{i}\right)=h_{\ell-1}\left(p_{i+1}\right)$. By consequence, $p_{i+1}$ makes its first appearance in the $\ell$ th row of $t^{\#}$ at position

$$
j+1=h_{\ell-1}\left(p_{i+1}\right)+1,
$$

${ }^{17}$ The columns of $t^{\#}$ in general are not contiguous, as in (6.10), but it does not matter. 
i.e., (6.11) holds for $p_{i+1}$. By iteration we have that (6.11) holds for all integers $p_{m}$ which are present in the $\ell$ th row of $t^{\#}$.

We can prove now that the columns of $t^{\#}$ are strictly increasing. More precisely, we prove the implication

$$
\ell<m \quad \text { and } \quad t_{\ell, j}^{\#} \geq t_{m, j^{\prime}}^{\#} \quad \Longrightarrow \quad j<j^{\prime} .
$$

Let, in fact, $s \leq \bar{s}$, and

$$
t_{\ell, j}^{\#}=\bar{s} \quad \text { and } \quad t_{m, j^{\prime}}^{\#}=s .
$$

From (6.11) it follows that

$$
j \leq h_{\ell}(\bar{s}) \quad \text { and } \quad j^{\prime} \geq h_{m-1}(s)+1 .
$$

Since $\widehat{\omega}$ is a lattice permutations, we get $h_{\ell}(\bar{s}) \leq h_{\ell}(s) \leq h_{m-1}(s)$, and (6.12) is proven.

Proof of (B) Using the definition $\tilde{t}_{i, j}:=t_{i, j-\alpha_{1}+\alpha_{i}}^{\#}$ and (6.12), we obtain that, for $\ell<m$,

$$
\tilde{t}_{\ell, j} \geq \tilde{t}_{m, j^{\prime}} \Longrightarrow j-\alpha_{1}+\alpha_{\ell}<j^{\prime}-\alpha_{1}+\alpha_{m} \quad \Longrightarrow \quad j<j^{\prime}
$$

Thus implication (6.12) holds for $\tilde{t}$. By consequence, the columns of $\tilde{t}$ (and obviously those of $t$ as well) are strictly increasing. This concludes the proof of Lemma 6.9 and Proposition 6.8. Hence Lemma 5.11 is also proven.

\section{References}

1. Aldous, D.: www.stat.berkeley.edu/ aldous/research/op/sgap.html

2. Biggs, N.: Algebraic Graph Theory, 2nd edn. Cambridge Mathematical Library. Cambridge University Press, Cambridge (1993)

3. Caputo, P., Liggett, T.M., Richthammer, T.: A recursive proof of Aldous' spectral gap conjecture. arXiv:0906.1238v3 (2009)

4. Chung, F.R.K.: Spectral Graph Theory. CBMS Regional Conference Series in Mathematics, vol. 92. American Mathematical Society, Providence (1997)

5. Curtis, C.W., Reiner, I.: Representation Theory of Finite Groups and Associative Algebras. Pure and Applied Mathematics, vol. XI. Interscience Publishers, a division of John Wiley \& Sons, New York (1962)

6. Diaconis, P., Shahshahani, M.: Generating a random permutation with random transpositions. Z. Wahrscheinlichkeitstheor. Verw. Geb. 57(2), 159-179 (1981)

7. Flatto, L., Odlyzko, A.M., Wales, D.B.: Random shuffles and group representations. Ann. Probab. 13(1), 154-178 (1985)

8. Friedman, J.: On Cayley graphs on the symmetric group generated by transpositions. Combinatorica 20(4), 505-519 (2000)

9. Godsil, C., Royle, G.: Algebraic Graph Theory. Graduate Texts in Mathematics, vol. 207. Springer, New York (2001)

10. Handjani, S., Jungreis, D.: Rate of convergence for shuffling cards by transpositions. J. Theor. Probab. 9(4), 983-993 (1996)

11. Ingram, R.E.: Some characters of the symmetric group. Proc. Am. Math. Soc. 1, 358-369 (1950)

12. James, G., Kerber, A.: The Representation Theory of the Symmetric Group. Encyclopedia of Mathematics and its Applications, vol. 16. Addison-Wesley, Reading (1981) 
13. Koma, T., Nachtergaele, B.: The spectral gap of the ferromagnetic $X X Z$ chain. Lett. Math. Phys. 40(1), 1-16 (1997)

14. Morris, B.: Spectral gap for the interchange process in a box. Electron. Commun. Probab. 13, 311-318 (2008)

15. Sagan, B.E.: The Symmetric Group: Representations, Combinatorial Algorithms, and Symmetric Functions, 2nd edn. Graduate Texts in Mathematics, vol. 203. Springer, New York (2001)

16. Starr, S., Conomos, M.: Asymptotics of the spectral gap for the interchange process on large hypercubes. arXiv:0802.1368v2 (2008) 